\title{
On classical and semiclassical properties of the Liouville theory with defects
}

\author{
Hasmik Poghosyan ${ }^{a}$ and Gor Sarkissian ${ }^{a, b}$ \\ ${ }^{a}$ Yerevan Physics Institute, \\ Alikhanian Br. 2, 0036 Yerevan, Armenia \\ ${ }^{b}$ Department of Physics, Yerevan State University, \\ Alex Manoogian 1, 0025 Yerevan, Armenia \\ E-mail: hasmikpoghos@gmail.com, gor.sarkissian@ysu.am
}

ABSTRACT: The Lagrangian of the Liouville theory with topological defects is analyzed in detail and general solution of the corresponding defect equations of motion is found. We study the heavy and light semiclassical limits of the defect two-point function found before via the bootstrap program. We show that the heavy asymptotic limit is given by the exponential of the Liouville action with defects, evaluated on the solutions with two singular points. We demonstrate that the light asymptotic limit is given by the finite dimensional path integral over solutions of the defect equations of motion with a vanishing energy-momentum tensor.

Keywords: Conformal Field Models in String Theory, D-branes, Bosonic Strings

ArXiv EPrint: 1505.00366 


\section{Contents}

1 Introduction 1

2 Classical Liouville theory with defects 3

2.1 Review of Liouville solution 3

2.2 Lagrangian of the Liouville theory with defect 6

2.3 Lagrangian of the Liouville theory with permutation branes 8

3 Permutation branes and defects in quantum Liouville $\quad 10$

$\begin{array}{lll}3.1 & \text { Review of quantum Liouville } & 10\end{array}$

$\begin{array}{lll}3.2 & \text { Permutation branes and defects in quantum Liouville } & 11\end{array}$

4 Semiclassical limits $\quad 12$

$\begin{array}{ll}4.1 \text { Heavy asymptotic limit } & 12\end{array}$

$\begin{array}{ll}4.2 \text { Light asymptotic limit } & 14\end{array}$

5 Defects in the light asymptotic limit $\quad 15$

6 Defects in the heavy asymptotic limit $\quad 20$

6.1 Heavy asymptotic limit of the correlation functions 20

$\begin{array}{ll}6.2 & \text { Evaluation of the action for classical solutions }\end{array}$

$\begin{array}{lll}7 & \text { Discussion } & 27\end{array}$

$\begin{array}{lr}\text { A Properties of } \Gamma \text { functions } & 28\end{array}$

$\begin{array}{ll}\text { B Volume form on the 3D hyperboloid } \mathrm{H}_{3}^{+} & 29\end{array}$

$\begin{array}{lr}\text { C Action evaluation } & 29\end{array}$

D Defect two-point function 33

\section{Introduction}

Defects in two-dimensional conformal field theories can be realized as oriented lines, separating different theories. We are interested in the special class of defects, for which the energy-momentum tensor is continuous across the defect [1]. Denoting the left- and rightmoving energy-momentum tensors of the two theories by $T^{(1)}, T^{(2)}$, and $\bar{T}^{(1)}, \bar{T}^{(2)}$, this condition takes the form:

$$
T^{(1)}=T^{(2)}, \quad \bar{T}^{(1)}=\bar{T}^{(2)} .
$$


Inserting a defect in the path integral is equivalent in the operator language to the insertion of an operator $D$ which maps the Hilbert space of CFT 1 to that of CFT 2. Condition (1.1) can be considered as implying that the corresponding operator $D$ commutes with the Virasoro modes:

$$
D L_{m}^{(1)}=L_{m}^{(2)} D \quad \text { and } \quad D \bar{L}_{m}^{(1)}=\bar{L}_{m}^{(2)} D .
$$

During the last few years topological defects in the Liouville and Toda field theories attracted some attention due to their relation to the Wilson lines in the AGT correspondence $[2-5] .{ }^{1}$ Defects in the Liouville field theory have been constructed in $[7,8]$. In these papers defects were constructed as operators on the Hilbert space of Liouville theory. To obtain these operators, two-point functions in the presence of defects were calculated using the conformal bootstrap program for defects, developed in $[7,9]$. It was shown in [7] that there are two families of defects: discrete, corresponding to the degenerate fields and labeled by a pair of positive integers $m$ and $n$, with eigenvalues

$$
\mathcal{D}_{m, n}(\alpha)=\frac{\sin \left(\pi m b^{-1}(2 \alpha-Q)\right) \sin (\pi n b(2 \alpha-Q))}{\sin \pi b^{-1}(2 \alpha-Q) \sin \pi b(2 \alpha-Q)},
$$

and continuous, labeled by one continuous parameter $s$ with eigenvalues

$$
\mathcal{D}_{s}(\alpha)=\frac{\cosh (2 \pi s(2 \alpha-Q))}{2 \sin \pi b^{-1}(2 \alpha-Q) \sin \pi b(2 \alpha-Q)} .
$$

We denoted here by $Q=b+\frac{1}{b}$ the background charge, and $\alpha$ labels primaries of Liouville theory. The defects of the discrete family have a one-dimensional world-volume, and in particular the identity defect $\mathcal{D}_{1,1}$ belongs to the discrete family. The defects of the continuous family have a two-dimensional world-volume. The details can be found in appendix D.

Recently also integrable defects were studied (see e.g. [10-18] ).

The Lagrangian for the continuous family of two-dimensional topological defects was suggested in [14]. It is demonstrated in [14] that topological defects are so called type-II defects, proposed in [12], allowing additional degrees of freedom associated with the defect itself. It is also shown in [14], that requiring the additional degrees of freedom to be represented by a holomorphic field, leads to the topological defects.

The aim of this work is to study correspondence between the continuous family of defects (1.4) and the one-parametric family of Lagrangians with defect proposed in [14].

First we find general solution of the defect equations of motion coming from the Lagrangian proposed in [14].

To link two-point functions in the presence of defects to the Lagrangian with defects we use two strategies: heavy and light asymptotic semiclassical limits [19, 20, 22-26]. In the light asymptotic limit we set $\alpha=\eta_{l} b$ and keep $\eta_{l}$ fixed for $b \rightarrow 0$, whereas in the heavy asymptotic limit we take $\alpha=\frac{\eta_{h}}{b}$ and hold $\eta_{h}$ fixed again for $b \rightarrow 0$.

These semiclassical limits were used in [20,22] to relate the quantum three-point functions in the Liouville and Toda theories with the corresponding classical actions. The heavy

\footnotetext{
${ }^{1}$ In fact in references $[2,3]$ the Verlinde loop operators are discussed, but they coincide with topological defects for the Cardy case [6].
} 
asymptotic limit plays an important role in the quantum uniformization program [21]. In papers [23-26] these techniques were generalized to the boundary Liouville and Toda theories. Both limits have recently proved to be very useful also to test AGT [27-30] and AdS/CFT correspondences [31-33].

The heavy and light asymptotic limits were reconsidered in [34] also for complex solutions of the analytically continued Liuoville theory.

Here we develop both procedures of the semiclassical limits to the Liouville theory with defects and find perfect agreement between the classical and bootstrap results. In particular we establish connection between the parameter $\kappa$ entering in the Lagrangian with defect and parameter $s$ labeling the defect operator (1.4):

$$
\kappa=\cosh (2 \pi s b)
$$

where it is understood that $s \rightarrow \infty$ and $b \rightarrow 0$ in a way that keeps $\sigma=s b$ fixed.

We show that in the light asymptotic limit the defect two-point functions can be obtained via the path integral over solutions of the defect equations of motion with vanishing energy-momentum tensor in the large $\sigma$ limit.

We demonstrate that in the heavy asymptotic limit defect two-point functions are given by the sum of exponentials of the action with defects evaluated on solutions with two singular points of the defect equations of motion. To understand better the semiclassical origin of the denominator in (1.4) in the heavy asymptotic limit, we consider analytic continuation of $\eta$ to the complex region in the spirit of [34]. We find a discrete family of solutions with two singular points, labelled by two integer numbers $N_{1}$ and $N_{2}$. But to fit to semiclassical limit of the defect two-point function and to have convergent series we should sum over the saddle points with nonnegative $N_{1}$ and $N_{2}$ for $\operatorname{Im}(2 \eta-1)>0$, and with nonpositive $N_{1}$ and $N_{2}$ if $\operatorname{Im}(2 \eta-1)<0$. This is an example of the Stokes phenomena [34-37].

The paper is organized in the following way.

In section 2 we analyze classical Liouville theory with defects. In subsection 2.1 we review the general solution of the Liouville equation. In subsection 2.2 we present general solution of the defect equations of motion. In subsection 2.3 we present the Lagrangian of the product of the Liouville theories on half-plane with the boundary condition specified by a permutation brane. In section 3 we review defects and permutation branes in quantum Liouville theory. In section 4 we review the heavy and light asymptotic semiclassical limits. In section 5 we calculate the defect two-point function in the light asymptotic limit. In section 6 we calculate the defect two-point function in the heavy asymptotic limit. In a series of appendices we describe some useful technical results.

\section{Classical Liouville theory with defects}

\subsection{Review of Liouville solution}

Let us recall some facts on classical Liouville theory.

The action of the Liouville theory is

$$
S=\frac{1}{2 \pi i} \int\left(\partial \phi \bar{\partial} \phi+\mu \pi e^{2 b \phi}\right) d^{2} z
$$

Here we use a complex coordinate $z=\tau+i \sigma$, and $d^{2} z \equiv d z \wedge d \bar{z}$ is the volume form. 
The field $\phi(z, \bar{z})$ satisfies the classical Liouville equation of motion

$$
\partial \bar{\partial} \phi=\pi \mu b e^{2 b \phi} .
$$

The general solution to (2.2), also derived below, was given by Liouville in terms of two arbitrary functions $A(z)$ and $B(\bar{z})[38]$

$$
\phi=\frac{1}{2 b} \log \left(\frac{1}{\pi \mu b^{2}} \frac{\partial A(z) \bar{\partial} B(\bar{z})}{(A(z)+B(\bar{z}))^{2}}\right) .
$$

The solution (2.3) is invariant if one transforms $A$ and $B$ simultaneously by the following constant Möbius transformations:

$$
A \rightarrow \frac{\zeta A+\beta}{\gamma A+\delta}, \quad B \rightarrow \frac{\zeta B-\beta}{-\gamma B+\delta}, \quad \zeta \delta-\beta \gamma=1 .
$$

Classical expressions for left and right components of the energy-momentum tensor are

$$
\begin{aligned}
& T=-(\partial \phi)^{2}+b^{-1} \partial^{2} \phi, \\
& \bar{T}=-(\bar{\partial} \phi)^{2}+b^{-1} \bar{\partial}^{2} \phi .
\end{aligned}
$$

Substituting (2.3) in (2.5) and (2.6) we get, that the components of the energy-momentum tensor are given by the Schwarzian derivatives of $A(z)$ and $B(\bar{z})$ :

$$
\begin{aligned}
& T=\{A ; z\}=\frac{1}{2 b^{2}}\left[\frac{A^{\prime \prime \prime}}{A^{\prime}}-\frac{3}{2} \frac{\left(A^{\prime \prime}\right)^{2}}{\left(A^{\prime}\right)^{2}}\right], \\
& \bar{T}=\{B ; \bar{z}\}=\frac{1}{2 b^{2}}\left[\frac{B^{\prime \prime \prime}}{B^{\prime}}-\frac{3}{2} \frac{\left(B^{\prime \prime}\right)^{2}}{\left(B^{\prime}\right)^{2}}\right] .
\end{aligned}
$$

The Schwarzian derivative is invariant under arbitrary constant Möbius transformation:

$$
\left\{\frac{\zeta F+\beta}{\gamma F+\delta} ; z\right\}=\{F ; z\}, \quad \zeta \delta-\beta \gamma=1 .
$$

Solutions of the Liouville equation (2.2) can be described also via linear combination of some holomorphic and anti-holomorphic functions. Let us introduce the function $V=e^{-b \phi}$. One can write the Liouville equation (2.2) as an equation for $V$

$$
V \partial \bar{\partial} V-\partial V \bar{\partial} V=-\pi \mu b^{2} .
$$

Also the left and right components of the energy-momentum tensor (2.5) and (2.6) can be written via $V$

$$
\begin{aligned}
& \partial^{2} V=-b^{2} V T, \\
& \bar{\partial}^{2} V=-b^{2} V \bar{T} .
\end{aligned}
$$

It is straightforward to check that the general solution of eq. (2.10) is given by linear combination of two holomorphic $a_{i}(z), i=1,2$, and two anti-holomorphic functions $b_{i}(\bar{z})$, $i=1,2$ :

$$
V=\sqrt{\pi \mu b^{2}}\left(a_{1}(z) b_{1}(\bar{z})-a_{2}(z) b_{2}(\bar{z})\right),
$$


satisfying the condition

$$
\left(a_{1} a_{2}^{\prime}-a_{1}^{\prime} a_{2}\right)\left(b_{1} b_{2}^{\prime}-b_{1}^{\prime} b_{2}\right)=1 .
$$

Usually the fields $a_{i}(z)$ and $b_{i}(\bar{z}), i=1,2$ are normalized to have the unit Wronskian:

$$
a_{1} a_{2}^{\prime}-a_{1}^{\prime} a_{2}=1
$$

and

$$
b_{1} b_{2}^{\prime}-b_{1}^{\prime} b_{2}=1 .
$$

It is easy to see that the left and right components of the energy-momentum tensor can be expressed via $a_{i}$ and $b_{i}$ in the very simple form:

$$
T=-\frac{1}{b^{2}} \frac{\partial^{2} a_{1}}{a_{1}}=-\frac{1}{b^{2}} \frac{\partial^{2} a_{2}}{a_{2}}
$$

and

$$
\bar{T}=-\frac{1}{b^{2}} \frac{\bar{\partial}^{2} b_{1}}{b_{1}}=-\frac{1}{b^{2}} \frac{\bar{\partial}^{2} b_{2}}{b_{2}} .
$$

The solutions (2.3) and (2.13) can be related in the following way. One can solve the unit Wronskian conditions (2.15) and (2.16) via a holomorphic $A(z)$ and an anti-holomorphic function $B(\bar{z})$

and

$$
a_{1}=\frac{1}{\sqrt{\partial A}} \quad \text { and } \quad a_{2}=\frac{A}{\sqrt{\partial A}}
$$

$$
b_{1}=\frac{B}{\sqrt{\bar{\partial} B}} \quad \text { and } \quad b_{2}=-\frac{1}{\sqrt{\bar{\partial} B}} .
$$

Inserting (2.19) and (2.20) in (2.13) we get (2.3). Note that the Möbius transformations of $A$ and $B(2.4)$ become linear $\mathrm{SL}(2, \mathbb{C})$ transformations of $a_{i}$ and $b_{i}$ :

$$
\begin{aligned}
& \tilde{a}_{1}=\delta a_{1}+\gamma a_{2}, \\
& \tilde{a}_{2}=\beta a_{1}+\zeta a_{2}
\end{aligned}
$$

and

$$
\begin{aligned}
& \tilde{b}_{1}=\zeta b_{1}+\beta b_{2}, \\
& \tilde{b}_{2}=\gamma b_{1}+\delta b_{2} .
\end{aligned}
$$

It is straightforward to check that indeed (2.13) is invariant under (2.21) and (2.22), and both of them keep the unit Wronskian condition.

One can also check, that both components of the energy-momentum tensor (2.17) and (2.18) are invariant under these transformations as well.

We finish this section with a remark which will be important in the parts of this work dealing with the light asymptotic limit. There we will consider an analytic continuation $\mu \rightarrow-\mu$. At this point it is convenient to write the solution (2.13) as:

$$
V=\sqrt{-\pi \mu b^{2}}\left(a_{1}(z) b_{1}(\bar{z})+a_{2}(z) b_{2}(\bar{z})\right) .
$$

It is easy to check that (2.23) also solves the Liouville equation, given that $a_{i}$ and $b_{i}, i=1,2$ obey the condition (2.14). 


\subsection{Lagrangian of the Liouville theory with defect}

Recently in [14] the action of the Liouville theory with topological defects was suggested:

$$
\begin{aligned}
& S^{\text {top }- \text { def }}=\frac{1}{2 \pi i} \int_{\Sigma_{1}}\left(\partial \phi_{1} \bar{\partial} \phi_{1}+\mu \pi e^{2 b \phi_{1}}\right) d^{2} z+\frac{1}{2 \pi i} \int_{\Sigma_{2}}\left(\partial \phi_{2} \bar{\partial} \phi_{2}+\mu \pi e^{2 b \phi_{2}}\right) d^{2} z \\
& +\int_{\partial \Sigma_{1}}\left[-\frac{1}{2 \pi} \phi_{2} \partial_{\tau} \phi_{1}+\frac{1}{2 \pi} \Lambda \partial_{\tau}\left(\phi_{1}-\phi_{2}\right)+\frac{\mu}{2} e^{\left(\phi_{1}+\phi_{2}-\Lambda\right) b}-\frac{1}{\pi b^{2}} e^{\Lambda b}\left(\cosh \left(\phi_{1}-\phi_{2}\right) b-\kappa\right)\right] \frac{d \tau}{i} .
\end{aligned}
$$

Here $\Sigma_{1}$ is the upper half-plane $\sigma=\operatorname{Im} z \geq 0$ and $\Sigma_{2}$ is the lower half-plane $\sigma=\operatorname{Im} z \leq$ 0 . The defect is located along their common boundary, which is the real axis $\sigma=0$ parametrized by $\tau=\operatorname{Re} z$. Note that $\Lambda(\tau)$ here is an additional field associated with the defect itself. The action (2.24) yields the following defect equations of motion at $\sigma=0$ :

$$
\begin{aligned}
\frac{1}{2 \pi}(\partial-\bar{\partial}) \phi_{1}+\frac{1}{2 \pi} \partial_{\tau} \phi_{2}-\frac{1}{2 \pi} \partial_{\tau} \Lambda+\frac{\mu b}{2} e^{\left(\phi_{1}+\phi_{2}-\Lambda\right) b}-\frac{1}{\pi b} e^{\Lambda b} \sinh \left(\phi_{1}-\phi_{2}\right) b & =0, \\
-\frac{1}{2 \pi}(\partial-\bar{\partial}) \phi_{2}-\frac{1}{2 \pi} \partial_{\tau} \phi_{1}+\frac{1}{2 \pi} \partial_{\tau} \Lambda+\frac{\mu b}{2} e^{\left(\phi_{1}+\phi_{2}-\Lambda\right) b}+\frac{1}{\pi b} e^{\Lambda b} \sinh \left(\phi_{1}-\phi_{2}\right) b & =0, \\
\frac{1}{2 \pi} \partial_{\tau}\left(\phi_{1}-\phi_{2}\right)-\frac{\mu b}{2} e^{\left(\phi_{1}+\phi_{2}-\Lambda\right) b}-\frac{1}{\pi b} e^{\Lambda b}\left(\cosh \left(\phi_{1}-\phi_{2}\right) b-\kappa\right) & =0 .
\end{aligned}
$$

The last equation is derived from variation of $\Lambda$.

Using that $\partial_{\tau}=\partial+\bar{\partial}$ and forming various linear combinations of equations (2.25)(2.27) we can bring them to the form:

$$
\begin{aligned}
\bar{\partial}\left(\phi_{1}-\phi_{2}\right) & =\pi \mu b e^{b\left(\phi_{1}+\phi_{2}\right)} e^{-\Lambda b} \\
\partial\left(\phi_{1}-\phi_{2}\right) & =\frac{2}{b} e^{\Lambda b}\left(\cosh \left(\phi_{1}-\phi_{2}\right) b-\kappa\right) . \\
\partial\left(\phi_{1}+\phi_{2}\right)-\partial_{\tau} \Lambda & =\frac{2}{b} e^{\Lambda b} \sinh \left(b\left(\phi_{1}-\phi_{2}\right)\right) .
\end{aligned}
$$

It is shown in [14] that requiring the defect equations of motion to hold for every $\sigma$ brings additionally to the condition, that $\Lambda$ is a restriction to the real axis of a holomorphic field

$$
\bar{\partial} \Lambda=0 .
$$

This condition allows to rewrite (2.30) in the form

$$
\partial\left(\phi_{1}+\phi_{2}-\Lambda\right)=\frac{2}{b} e^{\Lambda b} \sinh \left(b\left(\phi_{1}-\phi_{2}\right)\right) .
$$

It is checked in [14] that the system of the defect equations of motion (2.28)-(2.32) guarantees that both components of the energy-momentum tensor are continuous across the defects and therefore describes topological defects:

$$
\begin{aligned}
& -\left(\partial \phi_{1}\right)^{2}+b^{-1} \partial^{2} \phi_{1}=-\left(\partial \phi_{2}\right)^{2}+b^{-1} \partial^{2} \phi_{2}, \\
& -\left(\bar{\partial} \phi_{1}\right)^{2}+b^{-1} \bar{\partial}^{2} \phi_{1}=-\left(\bar{\partial} \phi_{2}\right)^{2}+b^{-1} \bar{\partial}^{2} \phi_{2} .
\end{aligned}
$$

Another interesting consequence of the defect equations of motion, found in [14], is the existence together with the holomorphic field $\Lambda(z)$ of an anti-holomorphic field $\Xi$ :

$$
\partial \Xi=0
$$


where

$$
\Xi=e^{-b\left(\phi_{1}+\phi_{2}\right)} e^{b \Lambda}\left(\cosh b\left(\phi_{1}-\phi_{2}\right)-\kappa\right)
$$

or alternatively

$$
\Xi=\frac{b}{2} e^{-b\left(\phi_{1}+\phi_{2}\right)} \partial\left(\phi_{1}-\phi_{2}\right) .
$$

Now we will present the general solution for defect equations of motion (2.28)-(2.32).

We will follow essentially the same strategy which was used in [39] for analyzing the boundary Liouville problem. On the one hand since the defect is topological both components of the energy-momentum tensor are equal being computed in terms of $\phi_{1}$ or $\phi_{2}$. On the other hand each component of the energy-momentum tensor is given by the Schwarzian derivative, which is invariant under the Möbius transformation. This naturally leads to the following solution:

$$
\begin{aligned}
\phi_{1} & =\frac{1}{2 b} \log \left(\frac{1}{\pi \mu b^{2}} \frac{\partial A \bar{\partial} B}{(A+B)^{2}}\right), \\
\phi_{2} & =\frac{1}{2 b} \log \left(\frac{1}{\pi \mu b^{2}} \frac{\partial C \bar{\partial} D}{(C+D)^{2}}\right),
\end{aligned}
$$

where

$$
C=\frac{\zeta A+\beta}{\gamma A+\delta} \quad \text { and } \quad D=\frac{\zeta^{\prime} B+\beta^{\prime}}{\gamma^{\prime} B+\delta^{\prime}} .
$$

Remembering that $\phi_{2}$ is invariant under the simultaneous Möbius transformation (2.4) of $C$ and $D$, we can set $B=D$. Therefore without loosing generality we can look for a solution in the form:

$$
\begin{aligned}
\phi_{1} & =\frac{1}{2 b} \log \left(\frac{1}{\pi \mu b^{2}} \frac{\partial A \bar{\partial} B}{(A+B)^{2}}\right), \\
\phi_{2} & =\frac{1}{2 b} \log \left(\frac{1}{\pi \mu b^{2}} \frac{\partial C \bar{\partial} B}{(C+B)^{2}}\right),
\end{aligned}
$$

where

$$
C=\frac{\zeta A+\beta}{\gamma A+\delta} .
$$

Substituting (2.41) and (2.42) in (2.28) we find that it is satisfied with

$$
e^{-\Lambda b}=\frac{A-C}{\sqrt{\partial A \partial C}} .
$$

Since $A$ and $C$ are holomorphic functions, $\Lambda$ is holomorphic as well, as it is stated in (2.31).

It is straightforward to check that (2.32) is satisfied as well with $\phi_{1}, \phi_{2}$ and $\Lambda$ given by (2.41), (2.42) and (2.44) respectively. And finally inserting (2.41), (2.42) and (2.44) in (2.29) we see that it is also fulfilled with

$$
\kappa=\frac{\zeta+\delta}{2} .
$$

Inserting (2.41), (2.42) in (2.37) one can check that

$$
\Xi=\frac{\pi \mu b^{2}}{2} \frac{\gamma B^{2}+B(\zeta-\delta)-\beta}{\bar{\partial} B} .
$$


Remembering that $B$ is anti-holomorphic we see that $\Xi$ is anti-holomorphic as well.

We can also write the solution of the defect equations of motion using solution of the Liouville equation in the form (2.13). Recalling that the Möbius transformations of the functions $A$ and $B$ become linear SL(2, $\mathbb{C})$ transformations of the functions $a_{i}$ and $b_{i}$, which leave the components of the energy-momentum tensor (2.17) and (2.18) invariant, we can write the solution (2.41)-(2.43) in the form:

$$
\begin{aligned}
& e^{-b \phi_{1}}=\sqrt{\pi \mu b^{2}}\left(a_{1}(z) b_{1}(\bar{z})-a_{2}(z) b_{2}(\bar{z})\right), \\
& e^{-b \phi_{2}}=\sqrt{\pi \mu b^{2}}\left(c_{1}(z) b_{1}(\bar{z})-c_{2}(z) b_{2}(\bar{z})\right),
\end{aligned}
$$

where denoting $\vec{a}=\left(a_{1}, a_{2}\right), \vec{c}=\left(c_{1}, c_{2}\right)$, and $D=\left(\begin{array}{ll}\delta & \gamma \\ \beta & \zeta\end{array}\right)$, one has

$$
\vec{c}=D \vec{a}
$$

and

$$
2 \kappa=\operatorname{Tr} D .
$$

At this point we would like to make the following remark. Let us consider the identity defect. It has $A=C$, and $\kappa=1$. Setting $A=C$ in (2.44) we obtain $e^{-\Lambda b}=0$. This result can be derived also directly setting $\phi_{1}=\phi_{2}$ in (2.28). Therefore the identity defect does not belong to the family of defects described by the action (2.24) and can be derived from them only in the limit $\Lambda \rightarrow \infty$. This can be understood recalling from appendix $\mathrm{D}$ that defects described by (2.24) have a two-dimensional world-volume in a sense that the values of $\phi_{1}(\tau)$ and $\phi_{2}(\tau)$ at an arbitrary point $\tau$ on the defect line are not constrained and the point $\left(\phi_{1}(\tau), \phi_{2}(\tau)\right)$ can take values in the whole plane $\mathbb{R}^{2}$. Contrary to this, the identity defect has a one-dimensional world-volume, since the point $\left(\phi_{1}(\tau), \phi_{2}(\tau)\right)$ takes values on one-dimensional diagonal $\phi_{1}=\phi_{2}$.

\subsection{Lagrangian of the Liouville theory with permutation branes}

We can also construct a folded version of the action (2.24) describing product of Liouville theories on a half-plane with boundary condition given by permutation branes:

$$
\begin{aligned}
& S^{\text {perm-brane }}=\frac{1}{2 \pi i} \int_{\Sigma}\left(\partial \phi_{1} \bar{\partial} \phi_{1}+\mu \pi e^{2 b \phi_{1}}+\partial \phi_{2} \bar{\partial} \phi_{2}+\mu \pi e^{2 b \phi_{2}}\right) d^{2} z \\
& +\int_{\partial \Sigma}\left[-\frac{1}{2 \pi} \phi_{2} \partial_{\tau} \phi_{1}+\frac{1}{2 \pi} \Lambda \partial_{\tau}\left(\phi_{1}-\phi_{2}\right)-\frac{\mu}{2} e^{\left(\phi_{1}+\phi_{2}-\Lambda\right) b}+\frac{1}{\pi b^{2}} e^{\Lambda b}\left(\cosh \left(\phi_{1}-\phi_{2}\right) b-\kappa\right)\right] \frac{d \tau}{i} .
\end{aligned}
$$

$\Sigma$ denotes here the upper half-plane $\sigma \geq 0$, and $\tau$ parameterizes the boundary located at $\sigma=0$. This action gives rise to the boundary equations

$$
\begin{aligned}
& \frac{1}{2 \pi}(\partial-\bar{\partial}) \phi_{1}+\frac{1}{2 \pi} \partial_{\tau} \phi_{2}-\frac{1}{2 \pi} \partial_{\tau} \Lambda-\frac{\mu b}{2} e^{\left(\phi_{1}+\phi_{2}-\Lambda\right) b}+\frac{1}{\pi b} e^{\Lambda b} \sinh \left(\phi_{1}-\phi_{2}\right) b=0 \\
& \frac{1}{2 \pi}(\partial-\bar{\partial}) \phi_{2}-\frac{1}{2 \pi} \partial_{\tau} \phi_{1}+\frac{1}{2 \pi} \partial_{\tau} \Lambda-\frac{\mu b}{2} e^{\left(\phi_{1}+\phi_{2}-\Lambda\right) b}-\frac{1}{\pi b} e^{\Lambda b} \sinh \left(\phi_{1}-\phi_{2}\right) b=0
\end{aligned}
$$




$$
\frac{1}{2 \pi} \partial_{\tau}\left(\phi_{1}-\phi_{2}\right)+\frac{\mu b}{2} e^{\left(\phi_{1}+\phi_{2}-\Lambda\right) b}+\frac{1}{\pi b} e^{\Lambda b}\left(\cosh \left(\phi_{1}-\phi_{2}\right) b-\kappa\right)=0 .
$$

Again using that $\partial_{\tau}=\partial+\bar{\partial}$ and forming various linear combinations, one can bring the system (2.52)-(2.54) to the form

$$
\begin{aligned}
\partial \phi_{2}-\bar{\partial} \phi_{1} & =\pi \mu b e^{b\left(\phi_{1}+\phi_{2}\right)} e^{-\Lambda b}, \\
\partial \phi_{1}-\bar{\partial} \phi_{2} & =-\frac{2}{b} e^{\Lambda b}\left(\cosh \left(\phi_{1}-\phi_{2}\right) b-\kappa\right), \\
\partial \phi_{1}+\bar{\partial} \phi_{2}-\partial_{\tau} \Lambda & =-\frac{2}{b} e^{\Lambda b} \sinh \left(b\left(\phi_{1}-\phi_{2}\right)\right) .
\end{aligned}
$$

One can check that equations (2.55)-(2.57) imply the permutation brane conditions:

$$
\begin{aligned}
& T^{(1)}=\left.\bar{T}^{(2)}\right|_{\sigma=0}, \\
& \bar{T}^{(1)}=\left.T^{(2)}\right|_{\sigma=0}
\end{aligned}
$$

or using (2.5) and (2.6)

$$
\begin{aligned}
& -\left(\partial \phi_{1}\right)^{2}+b^{-1} \partial^{2} \phi_{1}=-\left(\bar{\partial} \phi_{2}\right)^{2}+b^{-1} \bar{\partial}^{2} \phi_{2}, \\
& -\left(\bar{\partial} \phi_{1}\right)^{2}+b^{-1} \bar{\partial}^{2} \phi_{1}=-\left(\partial \phi_{2}\right)^{2}+b^{-1} \partial^{2} \phi_{2} .
\end{aligned}
$$

To solve equations (2.55)-(2.57) we will use the same strategy as before, with the only difference that now the Möbius transformation relates holomorphic and antiholomorphic functions:

$$
\begin{aligned}
& \phi_{1}=\frac{1}{2 b} \log \left(\frac{1}{\pi \mu b^{2}} \frac{\partial A \bar{\partial} B}{(A+B)^{2}}\right), \\
& \phi_{2}=\frac{1}{2 b} \log \left(\frac{1}{\pi \mu b^{2}} \frac{\partial B \bar{\partial} C}{(C+B)^{2}}\right),
\end{aligned}
$$

and

$$
C=\frac{\zeta A+\beta}{\gamma A+\delta}
$$

The expressions (2.61)-(2.63) solve equation (2.55) with the $\Lambda$ given by the relation

$$
e^{-\Lambda b}=\frac{C-A}{\sqrt{\partial A \bar{\partial} C}}
$$

It is straightforward to see that the expressions (2.61)-(2.63) together with the $\Lambda$ given by (2.64) solve also eq. (2.57).

Finally inserting $\phi_{1}, \phi_{2}$ and $\Lambda$ given by (2.61), (2.62) and (2.64) respectively in eq. (2.56) we get that it is satisfied as well with the following $\kappa$

$$
\kappa=\frac{\zeta+\delta}{2}
$$




\section{Permutation branes and defects in quantum Liouville}

\subsection{Review of quantum Liouville}

Liouville field theory is a conformal field theory enjoying the Virasoro algebra

$$
\left[L_{m}, L_{n}\right]=(m-n) L_{m+n}+\frac{c_{L}}{12}\left(n^{3}-n\right) \delta_{n,-m},
$$

with the central charge

$$
c_{L}=1+6 Q^{2} .
$$

Primary fields $V_{\alpha}$ in this theory, which are associated with exponential fields $e^{2 \alpha \varphi}$, have conformal dimensions

$$
\Delta_{\alpha}=\alpha(Q-\alpha) .
$$

The fields $V_{\alpha}$ and $V_{Q-\alpha}$ have the same conformal dimensions and represent the same primary field, i.e. they are proportional to each other:

$$
V_{\alpha}=S(\alpha) V_{Q-\alpha},
$$

with the reflection function

$$
S(\alpha)=\frac{\left(\pi \mu \gamma\left(b^{2}\right)\right)^{b^{-1}(Q-2 \alpha)}}{b^{2}} \frac{\Gamma(1-b(Q-2 \alpha)) \Gamma\left(-b^{-1}(Q-2 \alpha)\right)}{\Gamma(b(Q-2 \alpha)) \Gamma\left(1+b^{-1}(Q-2 \alpha)\right)} .
$$

Two-point functions of Liouville theory are given by the reflection function (3.5):

$$
\left\langle V_{\alpha}\left(z_{1}, \bar{z}_{1}\right) V_{\alpha}\left(z_{2}, \bar{z}_{2}\right)\right\rangle=\frac{S(\alpha)}{\left(z_{1}-z_{2}\right)^{2 \Delta_{\alpha}}\left(\bar{z}_{1}-\bar{z}_{2}\right)^{2 \Delta_{\alpha}}} .
$$

Introducing ZZ function [40]:

$$
W(\alpha)=-\frac{2^{3 / 4}\left(\pi \mu \gamma\left(b^{2}\right)\right)^{-\frac{(Q-2 \alpha)}{2 b}} \pi(Q-2 \alpha)}{\Gamma(1-b(Q-2 \alpha)) \Gamma\left(1-b^{-1}(Q-2 \alpha)\right)},
$$

the two-point function can be compactly written as

$$
S(\alpha)=\frac{W(Q-\alpha)}{W(\alpha)} .
$$

Another useful property of ZZ function is

$$
W(Q-\alpha) W(\alpha)=-2 \sqrt{2} \sin \pi b^{-1}(2 \alpha-Q) \sin \pi b(2 \alpha-Q) .
$$

The spectrum of the Liouville theory has the form

$$
\mathcal{H}=\int_{0}^{\infty} d P R_{\frac{Q}{2}+i P} \otimes R_{\frac{Q}{2}+i P}
$$

where $R_{\alpha}$ is the highest weight representation with respect to the Virasoro algebra. 


\subsection{Permutation branes and defects in quantum Liouville}

Let us recall the form of continuous family of defects and permutation branes in the Liouville field theory computed in $[7,8]$ using appropriate generalization of the Cardy-Lewellen equation [9]. The details can be found in appendix D. Topological defects are intertwining operators $X$ commuting with the Virasoro generators

$$
\left[L_{n}, X\right]=\left[\bar{L}_{n}, X\right]=0 .
$$

Such operators have the form

$$
X=\int_{\frac{Q}{2}+i \mathbb{R}} d \alpha \mathcal{D}(\alpha) \mathbb{P}^{\alpha}
$$

where $\mathbb{P}^{\alpha}$ are projectors on a subspace $R_{\alpha} \otimes R_{\alpha}$ :

$$
\mathbb{P}^{\alpha}=\sum_{N, M}(|\alpha, N\rangle \otimes \overline{|\alpha, M\rangle})(\langle\alpha, N| \otimes \overline{\langle\alpha, M|}) .
$$

Here $|\alpha, N\rangle$ and $\overline{|\alpha, M\rangle}$ are vectors of orthonormal bases of the left and right copies of $R_{\alpha}$ respectively. The eigenvalues $\mathcal{D}(\alpha)$ can be determined via the two-point functions computed in the presence of a defect $X$

$$
\left\langle V_{\alpha}\left(z_{1}, \bar{z}_{1}\right) X V_{\alpha}\left(z_{2}, \bar{z}_{2}\right)\right\rangle=\frac{\mathcal{D}(\alpha) S(\alpha)}{\left(z_{1}-z_{2}\right)^{2 \Delta_{\alpha}}\left(\bar{z}_{1}-\bar{z}_{2}\right)^{2 \Delta_{\alpha}}}
$$

It is shown in [7] that

$$
\left\langle V_{\alpha}\left(z_{1}, \bar{z}_{1}\right) X_{s} V_{\alpha}\left(z_{2}, \bar{z}_{2}\right)\right\rangle=-\frac{1}{W^{2}(\alpha)} \frac{2^{1 / 2} \cosh (2 \pi s(2 \alpha-Q))}{\left(z_{1}-z_{2}\right)^{2 \Delta_{\alpha}}\left(\bar{z}_{1}-\bar{z}_{2}\right)^{2 \Delta_{\alpha}}}
$$

and therefore for $D_{s}(\alpha)$ one can write using (3.8) and (3.9)

$$
\mathcal{D}_{s}(\alpha)=-\frac{2^{1 / 2} \cosh (2 \pi s(2 \alpha-Q))}{S(\alpha) W^{2}(\alpha)}=\frac{\cosh (2 \pi s(2 \alpha-Q))}{2 \sin \pi b^{-1}(2 \alpha-Q) \sin \pi b(2 \alpha-Q)} .
$$

The parameter $s$ is a continuous parameter labeling the defect. The defects can be characterized also by the value of the two-point function of the degenerate field $V_{-b / 2}$ in the presence of a defect. It is a function $A(b)$ of $b$. It is shown in [7] that the parameter $s$ is related to the function $A(b)$ by the equation:

$$
2 \cosh 2 \pi b s=A(b)\left(\frac{W(-b / 2)}{W(0)}\right)^{2} .
$$

The permutation branes boundary states $|B\rangle_{\mathcal{P}}$ on product $L_{1} \times L_{2}$ of two Liouville theories satisfy the gluing condition [41]:

$$
\begin{aligned}
\left(L_{n}^{(1)}-\bar{L}_{-n}^{(2)}\right)|B\rangle_{\mathcal{P}} & =0, \\
\left(L_{n}^{(2)}-\bar{L}_{-n}^{(1)}\right)|B\rangle_{\mathcal{P}} & =0 .
\end{aligned}
$$


Comparing the gluing conditions (3.18) and (3.11) one can see that topological defects related to permutation branes by folding trick, consisting of exchanging left and right components of the second copy, and hence these branes are characterized by the same two-point functions (3.15) with $z_{2}$ and $\bar{z}_{2}$ exchanged

$$
\left\langle V_{\alpha}^{(1)}\left(z_{1}, \bar{z}_{1}\right) V_{\alpha}^{(2)}\left(z_{2}, \bar{z}_{2}\right)\right\rangle_{\mathcal{P}}=-\frac{1}{W^{2}(\alpha)} \frac{2^{1 / 2} \cosh (2 \pi s(2 \alpha-Q))}{\left(z_{1}-\bar{z}_{2}\right)^{2 \Delta_{\alpha}}\left(\bar{z}_{1}-z_{2}\right)^{2 \Delta_{\alpha}}} .
$$

\section{Semiclassical limits}

\subsection{Heavy asymptotic limit}

Let us consider the action (2.1) for the rescaled variable $\varphi=2 b \phi$

$$
S=\frac{1}{8 \pi i b^{2}} \int\left(\partial \varphi \bar{\partial} \varphi+4 \lambda e^{\varphi}\right) d^{2} z
$$

where $\lambda=\pi \mu b^{2}$.

This form shows that $b^{2}$ plays in the Liouville theory the role of the Planck constant, and one can study semiclassical limit taking the limit $b \rightarrow 0$, in such a way that $\lambda$ is kept fixed.

Let us consider correlation functions in the path integral formalism:

$$
\left\langle V_{\alpha_{1}}\left(z_{1}, \bar{z}_{1}\right) \cdots V_{\alpha_{n}}\left(z_{n}, \bar{z}_{n}\right)\right\rangle=\int \mathcal{D} \varphi e^{-S} \prod_{i=1}^{n} \exp \left(\frac{\alpha_{i} \varphi\left(z_{i}, \bar{z}_{i}\right)}{b}\right) .
$$

We would like to calculate this integral in the semiclassical limit $b \rightarrow 0$ using the method of steepest descent, and we should decide how $\alpha_{i}$ scales with $b$. Since $S$ scales like $b^{-2}$, for operators to affect the saddle point, we should take $\alpha_{i}=\eta_{i} / b$, with $\eta_{i}$ fixed. The conformal weights $\Delta_{\alpha}=\eta(1-\eta) / b^{2}$ scale like $b^{-2}$ as well. This is the heavy asymptotic limit. Another choice of the operator scaling will be discussed in the next subsection.

We see from (4.2) that in the semiclasscial limit the correlation function is given by $e^{-S_{\mathrm{cl}}}$ where, at least naively, in a sense which will be clarified below, $S_{\mathrm{cl}}$ is the action

$$
S=\frac{1}{8 \pi i b^{2}} \int\left(\partial \varphi \bar{\partial} \varphi+4 \lambda e^{\varphi}\right) d^{2} z+\sum_{i=1}^{n} \frac{\eta_{i}}{b^{2}} \varphi\left(z_{i}, \bar{z}_{i}\right)
$$

evaluated on the solution of its equation of motion:

$$
\partial \bar{\partial} \varphi=2 \lambda e^{\varphi}-4 \pi \sum_{i=1}^{n} \eta_{i} \delta^{2}\left(z-z_{i}\right) .
$$

Assuming that in the vicinity of the insertion point $z_{i}$, one can ignore the exponential term we get that in the neighborhood of the point $z_{i} \varphi$ has the following behavior

$$
\varphi(z, \bar{z})=-4 \eta_{i} \log \left|z-z_{i}\right|+X_{i} \quad \text { as } \quad z \rightarrow z_{i} .
$$


One can insert this solution back into the equation of motion to check, if indeed the exponential term is subleading. We find, that this happens when

$$
\operatorname{Re} \eta_{i}<\frac{1}{2}
$$

This constraint is known as Seiberg bound [19]. It is the semiclassial version of the quantum condition (3.4) stating that $V_{\alpha}$ and $V_{Q-\alpha}$ represent the same quantum operator. Either $\alpha$ or $Q-\alpha$ always obey the Seiberg bound.

Remembering that in the Liouville theory we have also a background charge at infinity, conditions (4.5) should be complemented by the behavior at the infinity:

$$
\varphi(z, \bar{z})=-2 \log |z|^{2} \quad \text { as } \quad|z| \rightarrow \infty .
$$

Since the energy-momentum tensor in the presence of primary fields acquires a quadratic singularity,the functions $a_{j}, j=1,2$, should solve the equation

$$
\partial^{2} a_{j}+b^{2} T a_{j}=0
$$

where

$$
b^{2} T=\sum_{i=1}^{n}\left(\frac{\eta_{i}\left(1-\eta_{i}\right)}{\left(z-z_{i}\right)^{2}}+\frac{c_{i}}{\left(z-z_{i}\right)}\right)
$$

and $c_{i}$ are the so called accessory parameters.

If one tries naively to evaluate the action (4.3) on a solution obeying (4.5), one finds that it diverges. Therefore we should consider a regularized action. It was constructed in $[20]$ :

$$
\begin{aligned}
b^{2} S^{\mathrm{reg}}= & \frac{1}{8 \pi i} \int_{D-\cup_{i} d_{i}}\left(\partial \varphi \bar{\partial} \varphi+4 \lambda e^{\varphi}\right) d^{2} z+\frac{1}{2 \pi} \oint_{\partial D} \varphi d \theta+2 \log R \\
& -\sum_{i=1}^{n}\left(\frac{\eta_{i}}{2 \pi} \oint_{\partial d_{i}} \varphi d \theta_{i}+2 \eta_{i}^{2} \log \epsilon_{i}\right) .
\end{aligned}
$$

Here $D$ is a disc of radius $R, d_{i}$ is a disc of radius $\epsilon_{i}$ around $z_{i}$. It was shown in [20] that the action (4.10) satisfies the equation

$$
\frac{\partial}{\partial \eta_{i}} b^{2} S^{\mathrm{reg}}=-X_{i}
$$

where $X_{i}$ is defined by the boundary condition (4.5).

The Polyakov conjecture proved in [42] states, that the action (4.10) obeys also the relation:

$$
\frac{\partial}{\partial z_{i}} b^{2} S^{\mathrm{reg}}=-c_{i}
$$

Let us write down a regularized version of the action with a defect.

First of all let us write it in terms of $\lambda=\pi \mu b^{2}, \varphi_{1}=2 b \phi_{1}, \varphi_{2}=2 b \phi_{2}$, and $\tilde{\Lambda}=2 b \Lambda$ :

$$
b^{2} S^{\text {top-def }}=\frac{1}{8 \pi i} \int_{\Sigma_{1}}\left(\partial \varphi_{1} \bar{\partial} \varphi_{1}+4 \lambda e^{\varphi_{1}}\right) d^{2} z+\frac{1}{8 \pi i} \int_{\Sigma_{2}}\left(\partial \varphi_{2} \bar{\partial} \varphi_{2}+4 \lambda e^{\varphi_{2}}\right) d^{2} z
$$




$$
+\int_{\partial \Sigma_{1}}\left[-\frac{1}{8 \pi} \varphi_{2} \partial_{\tau} \varphi_{1}+\frac{1}{8 \pi} \tilde{\Lambda} \partial_{\tau}\left(\varphi_{1}-\varphi_{2}\right)+\frac{\lambda}{2 \pi} e^{\left(\varphi_{1}+\varphi_{2}-\tilde{\Lambda}\right) / 2}-\frac{1}{\pi} e^{\tilde{\Lambda} / 2}\left(\cosh \left(\frac{\varphi_{1}-\varphi_{2}}{2}\right)-\kappa\right)\right] \frac{d \tau}{i}
$$

Since we consider here only insertion of the bulk field, and do not consider insertion of the defect or boundary fields, the regularized action takes the form:

$$
\begin{aligned}
& b^{2} S^{\text {top }- \text { def }}=\frac{1}{8 \pi i} \int_{\Sigma_{1}^{R}-\cup_{i} d_{i}}\left(\partial \varphi_{1} \bar{\partial} \varphi_{1}+4 \lambda e^{\varphi_{1}}\right) d^{2} z \\
& -\sum_{i=1}^{n}\left(\frac{\eta_{i}}{2 \pi} \oint_{\partial d_{i}} \varphi_{1} d \theta_{i}+2 \eta_{i}^{2} \log \epsilon_{i}\right)+\frac{1}{2 \pi} \int_{s_{R}} \varphi_{1} d \theta+\log R \\
& +\frac{1}{8 \pi i} \int_{\Sigma_{2}^{R}-\cup_{j} d_{j}}\left(\partial \varphi_{2} \bar{\partial} \varphi_{2}+4 \lambda e^{\varphi_{2}}\right) d^{2} z \\
& -\sum_{j=n+1}^{n+m}\left(\frac{\eta_{j}}{2 \pi} \oint_{\partial d_{j}} \varphi_{2} d \theta_{j}+2 \eta_{j}^{2} \log \epsilon_{j}\right)+\frac{1}{2 \pi} \int_{s_{R_{2}}} \varphi_{2} d \theta+\log R \\
& +\int_{-R}^{R}\left[-\frac{1}{8 \pi} \varphi_{2} \partial_{\tau} \varphi_{1}+\frac{1}{8 \pi} \tilde{\Lambda} \partial_{\tau}\left(\varphi_{1}-\varphi_{2}\right)+\frac{\lambda}{2 \pi} e^{\left(\varphi_{1}+\varphi_{2}-\tilde{\Lambda}\right) / 2}-\frac{1}{\pi} e^{\tilde{\Lambda} / 2}\left(\cosh \left(\frac{\varphi_{1}-\varphi_{2}}{2}\right)-\kappa\right)\right] \frac{d \tau}{i},
\end{aligned}
$$

where $\Sigma_{i}^{R}$ is a half-disc of the radius $R$ and $s_{R i}$ is a semicircle of the radius $R$ in the half-plane $\Sigma_{i}, i=1,2$.

\subsection{Light asymptotic limit}

Another limit is the so called light asymptotic limit. Here we take

$$
\alpha=b \eta .
$$

In this limit the operator insertions have no influence and the components of the energymomentum tensor are (anti-) holomorphic and regular functions everywhere on sphere and hence vanish. Eq. (2.11) and (2.12) imply that $V \equiv e^{-b \phi}$ should be at most of first degree in $z$ and $\bar{z}$, thus leading to the solutions: ${ }^{2}$

$$
V(z, \bar{z} ; R)=\sqrt{-\lambda}(s z \bar{z}+t z+u \bar{z}+v), \quad R=\left(\begin{array}{cc}
s & t \\
u & v
\end{array}\right),
$$

where

$$
\operatorname{det} R=s v-u t=1 .
$$

Therefore the path integral in the light limit becomes a finite-dimensional integral over parameters $(s, t, u, v)$ which besides constraint (4.17) may satisfy some additional constraints like reality and defect/boundary condition. The reality of $V$ requires the matrix $R$ to be Hermitian. A way to parameterize the Hermitian matrices $R$ is

$$
R=\left(\begin{array}{cc}
X_{0}-X_{1} & X_{2}+i X_{3} \\
X_{2}-i X_{3} & X_{0}+X_{1}
\end{array}\right),
$$

\footnotetext{
${ }^{2}$ It is shown in [22] that to have solution in light limit one needs to perform analytical continuation $\mu \rightarrow-\mu$.
} 
where the constraint $X_{0}^{2}-X_{1}^{2}-X_{2}^{2}-X_{3}^{2}=1$, makes clear that the moduli space of the real solutions of the Liouville equation (2.2) with the vanishing energy-momentum tensor is a three-dimensional hyperboloid $H_{3}^{+}$. Hence, for example in the bulk Liouville theory,the correlation function in the light asymptotic limit takes the form

$$
\left\langle V_{b \eta_{1}}\left(z_{1}, \bar{z}_{1}\right) \cdots V_{b \eta_{n}}\left(z_{n}, \bar{z}_{n}\right)\right\rangle \rightarrow e^{-S_{l}} \int_{H_{3}^{+}} d R \prod_{i=1}^{n} V^{-2 \eta_{i}}\left(z_{i}, \bar{z}_{i} ; R\right),
$$

where $S_{l}$ is the value of the action on these solutions. The action $S_{l}$ is independent on $R$, since the derivative of $S_{l}$ by any element of $R$ vanishes, thanks to (4.16) being solution of the equations of motion:

$$
\frac{\partial S_{l}}{\partial R_{i j}}=\frac{\delta S_{l}}{\delta \phi} \frac{\partial \phi}{\partial R_{i j}}=0
$$

To avoid calculation of $S_{l}$ and some overall factors in the integration measure, it is more convenient, as suggested in [23], to compute the ratio

$$
\frac{\left\langle V_{b \eta_{1}}\left(z_{1}, \bar{z}_{1}\right) \cdots V_{b \eta_{n}}\left(z_{n}, \bar{z}_{n}\right)\right\rangle}{\left\langle V_{0}(0)\right\rangle} .
$$

Therefore defining

$$
\left\langle V_{b \eta_{1}}\left(z_{1}, \bar{z}_{1}\right) \cdots V_{b \eta_{n}}\left(z_{n}, \bar{z}_{n}\right)\right\rangle^{\text {light }} \equiv \int_{M} d R \prod_{i=1}^{n} V^{-2 \eta_{i}}\left(z_{i}, \bar{z}_{i} ; R\right),
$$

where $M$ is the moduli space of solutions with a vanishing energy-momentum tensor satisfying the corresponding boundary conditions in question, we can write

$$
\frac{\left\langle V_{b \eta_{1}}\left(z_{1}, \bar{z}_{1}\right) \cdots V_{b \eta_{n}}\left(z_{n}, \bar{z}_{n}\right)\right\rangle}{\left\langle V_{0}(0)\right\rangle} \rightarrow \frac{\left\langle V_{b \eta_{1}}\left(z_{1}, \bar{z}_{1}\right) \cdots V_{b \eta_{n}}\left(z_{n}, \bar{z}_{n}\right)\right\rangle^{\text {light }}}{\left\langle V_{0}(0)\right\rangle^{\text {light }}} .
$$

The moduli space $M$ for the Liouville theory with a boundary was studied in [23]. It was found that in the boundary Liouville problem $M$ is a subspace of $H_{3}^{+}$with $X_{3}$ set to the boundary cosmological constant. In the next section we will construct $M$ for the Liouville problem with defects.

\section{Defects in the light asymptotic limit}

Let us now specialize to the light asymptotic limit rules to the defects. We should find solutions for $\phi_{1}$ and $\phi_{2}$ in the form (4.16) satisfying the defect equations of motion. We find it convenient to use in this section a new constant $\tilde{\lambda} \equiv-\lambda=-\pi \mu b^{2}$. One can check that expressions

$$
\begin{aligned}
& V_{1}\left(z, \bar{z} ; R_{1}\right) \equiv e^{-b \phi_{1}}=\sqrt{\tilde{\lambda}}\left(s_{1} z \bar{z}+t_{1} z+u_{1} \bar{z}+v_{1}\right), \\
& R_{1}=\left(\begin{array}{ll}
s_{1} & t_{1} \\
u_{1} & v_{1}
\end{array}\right), \operatorname{det} R_{1}=1, R_{1}^{\dagger}=R_{1}
\end{aligned}
$$


and

$$
\begin{aligned}
& V_{2}\left(z, \bar{z} ; R_{2}\right) \equiv e^{-b \phi_{2}}=\sqrt{\tilde{\lambda}}\left(s_{2} z \bar{z}+t_{2} z+u_{2} \bar{z}+v_{2}\right), \\
& R_{2}=\left(\begin{array}{ll}
s_{2} & t_{2} \\
u_{2} & v_{2}
\end{array}\right), \operatorname{det} R_{2}=1, R_{2}^{\dagger}=R_{2}
\end{aligned}
$$

satisfy the defect equations of motion (2.28)-(2.32) with

$$
2 \kappa=\operatorname{Tr}\left(R_{2} R_{1}^{-1}\right)=s_{1} v_{2}+s_{2} v_{1}-u_{1} t_{2}-u_{2} t_{1}
$$

and

$$
e^{-b \Lambda}=z^{2}\left(s_{1} t_{2}-s_{2} t_{1}\right)+z\left(s_{1} v_{2}-s_{2} v_{1}+u_{1} t_{2}-u_{2} t_{1}\right)+u_{1} v_{2}-u_{2} v_{1} .
$$

Let us show that the relation (5.3) results from the general formula (2.50). Note that one can write the solution (5.1) in the general form (2.23)

$$
V_{1}\left(z, \bar{z} ; R_{1}\right)=\sqrt{\tilde{\lambda}}\left(s_{1} z \bar{z}+t_{1} z+u_{1} \bar{z}+v_{1}\right)=\sqrt{\tilde{\lambda}}\left[z\left(s_{1} \bar{z}+t_{1}\right)+\left(u_{1} \bar{z}+v_{1}\right)\right]
$$

with

$$
\begin{array}{ll}
a_{1}=z, & a_{2}=1, \\
b_{1}=s_{1} \bar{z}+t_{1}, & b_{2}=u_{1} \bar{z}+v_{1} .
\end{array}
$$

Remember that topological defects can be obtained in constructing $\phi_{2}$ by rotating the pair $a_{1}, a_{2}$ by a $\mathrm{SL}(2, \mathbb{C})$ matrix $D=\left(\begin{array}{l}\zeta \beta \\ \gamma \delta\end{array}\right)$, namely taking

$$
\begin{aligned}
& \tilde{a}_{1}=\zeta z+\beta, \\
& \tilde{a}_{2}=\gamma z+\delta
\end{aligned}
$$

and keeping the same $b_{1}$ and $b_{2}$ as in (5.6). Using (5.7) we get that $\phi_{2}$ is given by $R_{2}=D R_{1}$. Recalling that according to $(2.50) 2 \kappa=\operatorname{Tr} D$ we arrive to (5.3).

We would like to mention also a folded version of the defect solution, obeying the permutation brane boundary conditions. One can see that the expressions (5.1) and (5.2) satisfy the permutation branes boundary conditions (2.55)-(2.57) with

$$
2 \kappa=\operatorname{Tr}\left(R_{2}^{T} R_{1}^{-1}\right)=s_{1} v_{2}+s_{2} v_{1}-t_{1} t_{2}-u_{1} u_{2}
$$

and

$$
e^{-b \Lambda}=\tau^{2}\left(s_{2} t_{1}-s_{1} u_{2}\right)+\tau\left(s_{2} v_{1}-s_{1} v_{2}+t_{1} t_{2}-u_{1} u_{2}\right)+t_{2} v_{1}-u_{1} v_{2} .
$$

Note that equations (5.8) and (5.9) are in fact a folded version of the corresponding defect expressions (5.3) and (5.4) derived by exchanging $u_{2} \leftrightarrow t_{2}$, as a result of the $z_{2} \leftrightarrow \bar{z}_{2}$ exchange. The relation (5.8) can be justified again using the general formalism developed in section 2.3 .

In the parameterization (4.18) for the Hermitian matrices $R_{1}$ and $R_{2}$

$$
R_{1}=\left(\begin{array}{cc}
X_{0}-X_{1} & X_{2}+i X_{3} \\
X_{2}-i X_{3} & X_{0}+X_{1}
\end{array}\right), \quad R_{2}=\left(\begin{array}{cc}
Y_{0}-Y_{1} & Y_{2}+i Y_{3} \\
Y_{2}-i Y_{3} & Y_{0}+Y_{1}
\end{array}\right),
$$


the defect parameter (5.3) is equal to the Minkowski inner product of the vectors $X^{\mu}$ and $Y^{\mu}$

$$
\kappa=X^{\mu} Y_{\mu}=X_{0} Y_{0}-X_{1} Y_{1}-X_{2} Y_{2}-X_{3} Y_{3} .
$$

Using that $X_{0}, Y_{0} \geq 1$ and that $X^{\mu}$ and $Y^{\mu}$ both have the unit Minkowski norm, it is easy to show that $X^{\mu} Y_{\mu} \geq 1$, with equality when $X^{\mu}=Y^{\mu}$ [43]. It means that the real solutions of the defect equations of motion with vanishing energy-momentum tensor exist only for $\kappa \geq 1$. The border at $\kappa=1$ is expected. At this point $R_{1}=R_{2}$ and we have the identity defect which has $e^{-b \Lambda}=0$, which reflects that the identity defect does not belong to the family of two-dimensional defect described by the action (2.24). It may happen that the semiclassical limit for other values of $\kappa$ can be obtained using complex solutions of the defect equations of motion. Here we will consider only the values of $\kappa$ greater than 1 .

We are in a position to write the two-point correlation function in the presence of a defect:

$$
\begin{aligned}
& \left\langle V_{\alpha}\left(z_{1}, \bar{z}_{1}\right) X V_{\alpha}\left(z_{2}, \bar{z}_{2}\right)\right\rangle^{\text {light }}= \\
& =\int_{H_{3}^{+} \times H_{3}^{+}} d R_{1} d R_{2} \delta\left(\operatorname{Tr}\left(R_{2} R_{1}^{-1}\right)-2 \kappa\right) V_{1}^{-2 \eta}\left(z_{1}, \bar{z}_{1} ; R_{1}\right) V_{2}^{-2 \eta}\left(z_{2}, \bar{z}_{2} ; R_{2}\right) .
\end{aligned}
$$

Here $d R_{i}, i=1,2$ denotes integration measure on the 3D hyperboloid $H_{3}^{+}$. This expression allows to establish conformal invariance of a defect two-point function. Let us perform the transformation

$$
R_{1} \rightarrow L R_{1} L^{\dagger} \quad \text { and } \quad R_{2} \rightarrow L R_{2} L^{\dagger}
$$

where $L$ is a $\mathrm{SL}(2, C)$ matrix: $L=\left(\begin{array}{cc}m & n \\ k & l\end{array}\right)$. Note the transformation rule of the functions $V^{-2 \eta}(z, \bar{z} ; R)$ under $L$ :

$$
V^{-2 \eta}\left(z, \bar{z} ; L R L^{\dagger}\right)=\frac{1}{|n z+l|^{4 \eta}} V^{-2 \eta}\left(\frac{m z+k}{n z+l}, c . c ; R\right) .
$$

Performing the change of the integration variables (5.13), using that the $\delta$-function arguments is invariant under (5.13) the transformation rule (5.14), we obtain

$$
\begin{aligned}
& \left\langle V_{\alpha}\left(z_{1}, \bar{z}_{1}\right) X V_{\alpha}\left(z_{2}, \bar{z}_{2}\right)\right\rangle^{\text {light }}= \\
& =\frac{1}{\left|n z_{1}+l\right|^{4 \eta}} \frac{1}{\left|n z_{2}+l\right|^{4 \eta}}\left\langle V_{\alpha}\left(\frac{m z_{1}+k}{n z_{1}+l}, \text { c.c. }\right) X V_{\alpha}\left(\frac{m z_{2}+k}{n z_{2}+l}, \text { c.c. }\right)\right\rangle^{\text {light }},
\end{aligned}
$$

which is the standard consequence of the conformal invariance, when we remember that in the light asymptotic limit $\Delta_{\eta b} \rightarrow \eta$. This calculation shows that the invariance of the defect parameter $\kappa$ under (5.13) is related to the conformal invariance of the defect two-point function.

Using conformal invariance we can set $z_{1}$ to $\infty$ and $z_{2}$ to 0 to derive:

$$
\left\langle V_{b \eta}\left(z_{1}, \bar{z}_{1}\right) X V_{b \eta}\left(z_{2}, \bar{z}_{2}\right)\right\rangle^{\text {light }}=\frac{\tilde{\lambda}^{-2 \eta}}{\left(z_{1}-z_{2}\right)^{2 \eta}\left(\bar{z}_{1}-\bar{z}_{2}\right)^{2 \eta}}
$$




$$
\times \int_{H_{3}^{+} \times H_{3}^{+}} d R_{1} d R_{2} \delta\left(\operatorname{Tr}\left(R_{2} R_{1}^{-1}\right)-2 \kappa\right)\left(R_{1}\right)_{11}^{-2 \eta}\left(R_{2}\right)_{22}^{-2 \eta} .
$$

To calculate this integral we express the Hermitian matrices $R_{1}$ and $R_{2}$ as products

$$
R_{1}=g g^{\dagger}, \quad R_{2}=\tilde{g} \tilde{g}^{\dagger}, \quad g, \tilde{g} \in \mathrm{SL}(2, \mathbb{C}),
$$

implying that

$$
\begin{aligned}
& V_{1}=\sqrt{\tilde{\lambda}}\left(\left|g_{11} z+g_{21}\right|^{2}+\left|g_{12} z+g_{22}\right|^{2}\right), \\
& V_{2}=\sqrt{\tilde{\lambda}}\left(\left|\tilde{g}_{11} z+\tilde{g}_{21}\right|^{2}+\left|\tilde{g}_{12} z+\tilde{g}_{22}\right|^{2}\right) .
\end{aligned}
$$

At the next step we will parametrize $\tilde{g}$ as a product of matrices $g$ and $U$ :

$$
\tilde{g}=g U
$$

where $U$ is an $\mathrm{SL}(2, \mathbb{C})$ matrix

$$
U=\left(\begin{array}{ll}
u_{11} & u_{12} \\
u_{21} & u_{22}
\end{array}\right), \quad u_{11} u_{22}-u_{12} u_{21}=1 .
$$

Inserting (5.17) and (5.20) in (5.3) we obtain

$$
2 \kappa=\operatorname{Tr} U U^{\dagger} .
$$

This can be understood noting that the solutions (5.18) and (5.19) correspond to

$$
\begin{aligned}
& a_{i}(z)=g_{1 i} z+g_{2 i}, \quad \tilde{a}_{i}(z)=\tilde{g}_{1 i} z+\tilde{g}_{2 i}, \quad i=1,2, \\
& b_{i}(\bar{z})=\bar{g}_{1 i} \bar{z}+\bar{g}_{2 i}, \quad \tilde{b}_{i}(\bar{z})=\overline{\tilde{g}}_{1 i} \bar{z}+\overline{\tilde{g}}_{2 i}, \quad i=1,2 .
\end{aligned}
$$

It is obvious that

$$
\begin{aligned}
& \tilde{a}_{i}=\sum_{j=1}^{2} a_{j} u_{j i}, \\
& \tilde{b}_{i}=\sum_{j=1}^{2} b_{j} \bar{u}_{j i} .
\end{aligned}
$$

We see that passing from $g$ to $\tilde{g}=g U$ brings to the simultaneous rotations of $a_{i}$ and $b_{i}$, $i=1,2$, by matrices $U$ and $\bar{U}$. Therefore the defect parameter $\kappa$ is equal to the trace of the product $U U^{\dagger}$. In these variables the integral (5.16) simplifies and reads

$$
\begin{aligned}
& \left\langle V_{b \eta}\left(z_{1}, \bar{z}_{1}\right) X V_{b \eta}\left(z_{2}, \bar{z}_{2}\right)\right\rangle^{\text {light }}=\frac{\tilde{\lambda}^{-2 \eta}}{\left(z_{1}-z_{2}\right)^{2 \eta}\left(\bar{z}_{1}-\bar{z}_{2}\right)^{2 \eta}} \\
& \times \int d R_{1} d U \delta\left(\left|u_{11}\right|^{2}+\left|u_{12}\right|^{2}+\left|u_{21}\right|^{2}+\left|u_{22}\right|^{2}-2 \kappa\right)\left(R_{1}\right)_{11}^{-2 \eta}\left(R_{2}\right)_{22}^{-2 \eta},
\end{aligned}
$$

where $d R_{1}$ and $d U$ are the corresponding integration measures which will be elaborated below. 
Using $\mathrm{SU}(2)$ freedom in the choice of $g$ we can adopt the parameterization

$$
g=\left(\begin{array}{cc}
\rho_{1}^{-1} & w_{1} \\
0 & \rho_{1}
\end{array}\right), \quad \rho_{1} \in \mathbb{R}, \quad w_{1} \in \mathbb{C}
$$

and

$$
R_{1}=\left(\begin{array}{cc}
\rho_{1}^{-2}+\left|w_{1}\right|^{2} & \rho_{1} w_{1} \\
\rho_{1} \bar{w}_{1} & \rho_{1}^{2}
\end{array}\right)
$$

Parameterizing $\tilde{g}$ in the same way

$$
\tilde{g}=\left(\begin{array}{cc}
\rho_{2}^{-1} & w_{2} \\
0 & \rho_{2}
\end{array}\right), \quad \rho_{2} \in \mathbb{R}, \quad w_{2} \in \mathbb{C},
$$

we find that the elements of the matrix $U=g^{-1} \tilde{g}$ satisfy the relations:

$$
\begin{aligned}
u_{21} & =0, \\
u_{22} & =u_{11}^{-1} \equiv u, \quad u \in \mathbb{R}, \\
\rho_{2} & =\rho_{1} u, \\
w_{2} & =\rho_{1}^{-1} u_{12}+w_{1} u .
\end{aligned}
$$

Eq. (5.31) implies

$$
R_{2}=\left(\begin{array}{cc}
\rho_{1}^{-2} u^{-2}+\left|\rho_{1}^{-1} u_{12}+w_{1} u\right|^{2} & \rho_{1} u\left(\rho_{1}^{-1} u_{12}+w_{1} u\right) \\
\rho_{1} u\left(\rho_{1}^{-1} \bar{u}_{12}+\bar{w}_{1} u\right) & \rho_{1}^{2} u^{2}
\end{array}\right)
$$

Using the volume form on the $3 \mathrm{D}$ hyperboloid $\mathrm{H}_{3}^{+}$computed in appendix B (B.5), one obtains for the integration measure

$$
d R_{1} d R_{2}=4 \rho_{1} d \rho_{1} d^{2} w_{1} u d u d^{2} u_{12} .
$$

Now the integral (5.27) takes the form

$$
\begin{aligned}
& \left\langle V_{b \eta}\left(z_{1}, \bar{z}_{1}\right) X V_{b \eta}\left(z_{2}, \bar{z}_{2}\right)\right\rangle^{l i g h t}=\frac{4 \tilde{\lambda}^{-2 \eta}}{\left(z_{1}-z_{2}\right)^{2 \eta}\left(\bar{z}_{1}-\bar{z}_{2}\right)^{2 \eta}} \\
& \times \int \rho_{1} d \rho_{1} d^{2} w_{1} u d u d^{2} u_{12} \delta\left(u^{2}+\frac{1}{u^{2}}+\left|u_{12}\right|^{2}-2 \kappa\right) \frac{1}{\left(\rho_{1}^{-2}+\left|w_{1}\right|^{2}\right)^{2 \eta}} \frac{1}{\rho_{1}^{4 \eta} u^{4 \eta}} .
\end{aligned}
$$

We see that the delta function in the integrand of (5.35) can be different from zero only for $\kappa>1$ in agreement with discussion after formula (5.11). Performing the integral over $u_{12}$ and then over $u$ we obtain

$$
\begin{aligned}
& \left\langle V_{b \eta}\left(z_{1}, \bar{z}_{1}\right) X V_{b \eta}\left(z_{2}, \bar{z}_{2}\right)\right\rangle^{\text {light }}= \\
& =2 \pi \tilde{\lambda}^{-2 \eta} \frac{\left(\left(\kappa+\sqrt{\kappa^{2}-1}\right)^{1-2 \eta}-\left(\kappa-\sqrt{\kappa^{2}-1}\right)^{1-2 \eta}\right)}{(1-2 \eta)\left(z_{1}-z_{2}\right)^{2 \eta}\left(\bar{z}_{1}-\bar{z}_{2}\right)^{2 \eta}} \\
& \times \int \rho_{1} d \rho_{1} d^{2} w_{1} \frac{1}{\left(\rho_{1}^{-2}+\left|w_{1}\right|^{2}\right)^{2 \eta}} \frac{1}{\rho_{1}^{4 \eta}} .
\end{aligned}
$$


Performing the integral over $w_{1}$ one gets

$$
\int \rho_{1} d \rho_{1} d^{2} w_{1} \frac{1}{\left(\rho_{1}^{-2}+\left|w_{1}\right|^{2}\right)^{2 \eta}} \frac{1}{\rho_{1}^{4 \eta}}=\frac{1}{2 \eta-1} \int \frac{d \rho_{1}}{\rho_{1}}=\frac{1}{2 \eta-1} \delta(0) .
$$

The integral with respect to $w_{1}$ converges if $2 \eta>1$. Having computed the integral under this assumption, we can define it away from this region by analytic continuation. The integral with respect to $\rho_{1}$ diverges. This divergence was analyzed in [19] and related to the infinite volume of the dilation group. It gives rise in fact to the $\delta(0)$ which appears in the two-point function of coincident fields of the continuous spectrum. We get a finite result taking the ratio

$$
\frac{\left\langle V_{b \eta}\left(z_{1}, \bar{z}_{1}\right) X V_{b \eta}\left(z_{2}, \bar{z}_{2}\right)\right\rangle^{\text {light }}}{\left\langle V_{0}\left(z_{1}, \bar{z}_{1}\right) X V_{0}\left(z_{2}, \bar{z}_{2}\right)\right\rangle^{\text {light }}}=\frac{\tilde{\lambda}^{-2 \eta} \sinh 2 \pi \sigma(1-2 \eta)}{(1-2 \eta)^{2}\left(z_{1}-z_{2}\right)^{2 \eta}\left(\bar{z}_{1}-\bar{z}_{2}\right)^{2 \eta} \sinh 2 \pi \sigma} .
$$

Here we set $\kappa=\cosh 2 \pi \sigma$.

Using the properties of the $\Gamma$ functions collected in appendix A one can calculate the light asymptotic limit of the ZZ function (3.7):

$$
\frac{W_{\alpha=\eta b}^{-1}}{W_{\alpha=0}^{-1}} \rightarrow \tilde{\lambda}^{-\eta} \frac{1}{1-2 \eta},
$$

and setting $s=\frac{\sigma}{b}$ and $\alpha=\eta b$ we also obtain

$$
\frac{\cosh 2 \pi s(2 \alpha-Q)}{\cosh 2 \pi s Q} \rightarrow e^{-4 \pi \eta|\sigma|} .
$$

Hence, recalling (3.15) we get in the light asymptotic limit for the defect two-point function derived via the bootstrap program

$$
\frac{\left\langle V_{b \eta}\left(z_{1}, \bar{z}_{1}\right) X V_{b \eta}\left(z_{2}, \bar{z}_{2}\right)\right\rangle}{\left\langle V_{0}\left(z_{1}, \bar{z}_{1}\right) X V_{0}\left(z_{2}, \bar{z}_{2}\right)\right\rangle} \rightarrow \frac{\tilde{\lambda}^{-2 \eta}}{(2 \eta-1)^{2}} \frac{e^{-4 \pi \eta|\sigma|}}{\left(z_{1}-z_{2}\right)^{2 \eta}\left(\bar{z}_{1}-\bar{z}_{2}\right)^{2 \eta}} .
$$

In the limit of large $\sigma$ we get full agreement between (5.38) and (5.41). It may happen that inclusion of one-loop determinants could make this agreement exact for all values of $\sigma$. The study of this point is left for future work. It is interesting to note, that in boundary conformal Toda field theory the agreement between the light asymptotic limit of boundary one-point function with the path integral calculations was also reached in [23] in the limit of the large boundary cosmological constant.

\section{Defects in the heavy asymptotic limit}

\subsection{Heavy asymptotic limit of the correlation functions}

In this section we consider the heavy asymptotic limit of two-point functions in the presence of defects (3.15). Now we should find asymptotic behaviour of the inverse ZZ function (3.7) and of the factor $\cosh (2 \pi s(2 \alpha-Q))$ in the limit $b \rightarrow 0$, setting $\alpha=\frac{\eta}{b}$, and $s=\frac{\sigma}{b}$. In the heavy asymptotic limit we should keep only terms having the form $\sim e^{1 / b^{2}}$. 
To understand the semiclassical origin of the denominator in (3.16) we find very useful to consider, in the spirit of [34], analytic continuation of the Liouville theory with a complex $\eta$ and complex saddle points.

Taking $\eta$ to satisfy the Seiberg bound (4.6) $\operatorname{Re} \eta<\frac{1}{2}$, and using properties of $\Gamma$ functions collected in appendix A, we obtain

$$
W_{\alpha=\frac{\eta}{b}}^{-1} \rightarrow C(b, \eta) \lambda^{\frac{1-2 \eta}{2 b^{2}}} \frac{1}{\sin \pi\left(\frac{2 \eta-1}{b^{2}}\right)} \exp \left(\frac{2 \eta-1}{b^{2}}[\log (1-2 \eta)-1]\right) .
$$

where

$$
\begin{aligned}
C(b, \eta) & =-\frac{2^{-3 / 4} b^{3} \Gamma(2 \eta)}{(2 \eta-1)^{2}} \\
& =\exp \left(-\frac{3}{4} \log 2+i \pi+\log \Gamma(2 \eta)-2 \log (1-2 \eta)+3 \log b\right)
\end{aligned}
$$

We see that all the terms in (6.2) are negligible compare to terms growing like $\sim e^{1 / b^{2}}$ in the limit $b \rightarrow 0$, and therefore $C(b, \eta)$ can be omitted. The importance of the term $\frac{1}{\sin \pi\left(\frac{2 \eta-1}{b^{2}}\right)}$ is explained in [34]. It was shown that this term in the semiclassical interpretation arises as a sum over some "instanton" like sectors. As a preparation to this point we will expand this term in two ways as suggested in [34]. Denoting $y=e^{i \pi(2 \eta-1) / b^{2}}$ one can write

$$
\frac{1}{\sin \pi\left(\frac{2 \eta-1}{b^{2}}\right)}=\frac{2 i}{y-y^{-1}}=2 i \sum_{k=0}^{\infty} y^{-(2 k+1)}=-2 i \sum_{k=0}^{\infty} y^{2 k+1} .
$$

One expansion is valid for $|y|>1$ and one for $|y|<1$. So either way, there is a set $T$ of integers with

$$
\frac{1}{\sin \pi\left(\frac{2 \eta-1}{b^{2}}\right)}= \pm 2 i \sum_{M \in T} e^{2 i \pi(M \mp 1 / 2)(2 \eta-1) / b^{2}}
$$

where $T$ consists of nonnegative integers if $\operatorname{Im}(2 \eta-1) / b^{2}>0$ and of nonpositive ones if $\operatorname{Im}(2 \eta-1) / b^{2}<0$.

The set $T$ in (6.4) can be understood as sum of saddle points in the minisuperspace approximation keeping only constant mode of $\phi$. In this approximation the Liouville path integral becomes the integral representation of the $\Gamma(x)$ function [34]:

$$
\Gamma(x)=\int_{-\infty}^{\infty} d \phi \exp (-S)
$$

where the minisuperspace action is

$$
S=-x \phi+e^{\phi}
$$

The steepest descent analysis of the $\Gamma(x)$ function asymptotic behaviour for the large negative $x$, was carried out in [44]. It is based on the lengthy and careful analysis of the integration contours of the integral representation of the $\Gamma(x)$ function (6.5), along which 
it converges in quadrants $\operatorname{Re} x<0, \operatorname{Im} x>0$ and $\operatorname{Re} x<0, \operatorname{Im} x<0$. In the physical literature it is reviewed in $[34,37]$. In this way we obtain the factor $\frac{1}{\sin \pi x}$ in (A.2) as a sum over the saddle points of the action (6.6).

Setting $\alpha=\frac{\eta}{b}$ and $s=\frac{\sigma}{b}$ we easily obtain:

$$
\cosh 2 \pi s(2 \alpha-Q) \rightarrow e^{\frac{2 \pi}{b^{2}}|\sigma|(1-2 \eta)} .
$$

Now we are in a position to write down the limiting form of the defects correlation functions.

Inserting (6.1), (6.7) in (3.15) we can write in the heavy asymptotic limit

$$
\begin{aligned}
& \left\langle V_{\alpha}\left(z_{1}, \bar{z}_{1}\right) X V_{\alpha}\left(z_{2}, \bar{z}_{2}\right)\right\rangle \sim\left(z_{1}-z_{2}\right)^{-2 \eta(1-\eta) / b^{2}}\left(\bar{z}_{1}-\bar{z}_{2}\right)^{-2 \eta(1-\eta) / b^{2}} \\
& \times \lambda^{\frac{1-2 \eta}{b^{2}}} \frac{1}{\sin ^{2} \pi\left(\frac{2 \eta-1}{b^{2}}\right)} \exp \left(\frac{4 \eta-2}{b^{2}}[\log (1-2 \eta)-1]\right) e^{\frac{2 \pi}{b^{2}}|\sigma|(1-2 \eta)} .
\end{aligned}
$$

Using also (6.4) we get

$$
\left\langle V_{\alpha}\left(z_{1}, \bar{z}_{1}\right) X V_{\alpha}\left(z_{2}, \bar{z}_{2}\right)\right\rangle \sim \sum_{M_{1}, M_{2} \in T} \exp \left(-S_{M_{1}, M_{2}}^{\text {def }}\right)
$$

where

$$
\begin{aligned}
b^{2} S_{M_{1}, M_{2}}^{\mathrm{def}}= & -2 i \pi\left(M_{1}+M_{2} \mp 1\right)(2 \eta-1)+4 \eta(1-\eta) \log \left|z_{1}-z_{2}\right| \\
& -(1-2 \eta) \log \lambda-(4 \eta-2) \log (1-2 \eta)+(4 \eta-2)-2 \pi|\sigma|(1-2 \eta) .
\end{aligned}
$$

It is instructive to compare the heavy asymptotic limit of the defect two-point function with the corresponding limit of the usual two-point function, computed in [34]

$$
\begin{aligned}
& \left\langle V_{\alpha}\left(z_{1}, \bar{z}_{1}\right) V_{\alpha}\left(z_{2}, \bar{z}_{2}\right)\right\rangle \sim\left|z_{1}-z_{2}\right|^{-4 \eta(1-\eta) / b^{2}} \\
& \times \lambda^{(1-2 \eta) / b^{2}} \frac{1}{\sin \pi(2 \eta-1) / b^{2}} \exp \left(\frac{4 \eta-2}{b^{2}}[\log (1-2 \eta)-1]\right) .
\end{aligned}
$$

The relation of (6.8) to (6.11) naturally gives the heavy asymptotic limit of the eigenvalues $\mathcal{D}_{s}(\alpha)$ of the defect operator:

$$
\mathcal{D}_{s}(\alpha)=\frac{\left\langle V_{\alpha}\left(z_{1}, \bar{z}_{1}\right) X V_{\alpha}\left(z_{2}, \bar{z}_{2}\right)\right\rangle}{\left\langle V_{\alpha}\left(z_{1}, \bar{z}_{1}\right) V_{\alpha}\left(z_{2}, \bar{z}_{2}\right)\right\rangle} \rightarrow \frac{e^{\frac{2 \pi}{b^{2}}|\sigma|(1-2 \eta)}}{\sin \pi\left(\frac{2 \eta-1}{b^{2}}\right)} .
$$

Of course (6.12) can be also easily derived directly from (3.16) in the heavy asymptotic limit.

\subsection{Evaluation of the action for classical solutions}

According to the general prescription of the semiclassical heavy asymptotic limit, we should find solutions of the Liouville equation, satisfying the defect equations of motion and possessing the logarithmic singularities (4.5) at points $z_{1}$ and $z_{2}$. The form of the solution of the defect equations of motion (2.41) and (2.42) implies that we should find functions 
$A(z), C(z)$ and $B(\bar{z})$ in such a way that $\phi_{1}$ has a logarithmic singularity at the point $z_{1}$ and $\phi_{2}$ has a logarithmic singularity at the point $z_{2}$. Since the energy-momentum tensor is continuous across a defect this implies that we should find solutions possessing two singular points. Two-point solutions are well known ( see for example [34]) and we can build from them the Ansatz satisfying the defect equations of motion.

To build the solution with the required singularities one should take a function $A(z)$ which is smooth and holomorphic away from $z_{1}$ and $z_{2}$. Let us take $A(z)$ as

$$
A(z)=e^{2 \nu_{1}}\left(z-z_{1}\right)^{2 \eta-1}\left(z-z_{2}\right)^{1-2 \eta} .
$$

One has also

$$
\begin{aligned}
& a_{1}=\frac{1}{\sqrt{\partial A}}=\frac{e^{-\nu_{1}}}{\sqrt{\left(z_{1}-z_{2}\right)(2 \eta-1)}}\left(z-z_{1}\right)^{1-\eta}\left(z-z_{2}\right)^{\eta}, \\
& a_{2}=\frac{A}{\sqrt{\partial A}}=\frac{e^{\nu_{1}}}{\sqrt{\left(z_{1}-z_{2}\right)(2 \eta-1)}}\left(z-z_{1}\right)^{\eta}\left(z-z_{2}\right)^{1-\eta} .
\end{aligned}
$$

Inserting (6.14) or (6.15) in (2.17) we obtain the energy-momentum tensor

$$
b^{2} T=\frac{\eta(1-\eta)}{\left(z-z_{1}\right)^{2}}+\frac{\eta(1-\eta)}{\left(z-z_{2}\right)^{2}}-\frac{2 \eta(1-\eta)}{z_{1}-z_{2}}\left(\frac{1}{z-z_{1}}-\frac{1}{z-z_{2}}\right),
$$

which indeed possesses two singular points (4.9), with accessory parameters

$$
c_{2}=-c_{1}=\frac{2 \eta(1-\eta)}{z_{1}-z_{2}} .
$$

The anti-holomorphic part is:

$$
\begin{aligned}
B(\bar{z}) & =-\left(\bar{z}-\bar{z}_{1}\right)^{1-2 \eta}\left(\bar{z}-\bar{z}_{2}\right)^{2 \eta-1} \\
b_{1} & =\frac{B}{\sqrt{\bar{\partial} B}}=\frac{1}{\sqrt{\left(\bar{z}_{1}-\bar{z}_{2}\right)(2 \eta-1)}}\left(\bar{z}-\bar{z}_{1}\right)^{1-\eta}\left(\bar{z}-\bar{z}_{2}\right)^{\eta} \\
b_{2} & =-\frac{1}{\sqrt{\bar{\partial} B}}=\frac{1}{\sqrt{\left(\bar{z}_{1}-\bar{z}_{2}\right)(2 \eta-1)}}\left(\bar{z}-\bar{z}_{1}\right)^{\eta}\left(\bar{z}-\bar{z}_{2}\right)^{1-\eta} .
\end{aligned}
$$

Let us take the holomorphic part for $\phi_{2}$ as

$$
C(z)=e^{2 \nu_{2}}\left(z-z_{1}\right)^{2 \eta-1}\left(z-z_{2}\right)^{1-2 \eta}=e^{2\left(\nu_{2}-\nu_{1}\right)} A(z),
$$

and the antiholomorphic part again given by (6.18). Using (2.45) one gets

$$
\kappa=\cosh \left(\nu_{2}-\nu_{1}\right) .
$$

Inserting (6.13), (6.21) and (6.18) in (2.41) and (2.42) we obtain:

$$
\begin{gathered}
e^{-\varphi_{1}}=\frac{\lambda}{(2 \eta-1)^{2}\left|z_{1}-z_{2}\right|^{2}}\left(e^{\nu_{1}}\left|z-z_{1}\right|^{2 \eta}\left|z-z_{2}\right|^{2-2 \eta}\right. \\
\left.-e^{-\nu_{1}}\left|z-z_{1}\right|^{2-2 \eta}\left|z-z_{2}\right|^{2 \eta}\right)^{2}
\end{gathered}
$$




$$
\begin{gathered}
e^{-\varphi_{2}}=\frac{\lambda}{(2 \eta-1)^{2}\left|z_{1}-z_{2}\right|^{2}}\left(e^{\nu_{2}}\left|z-z_{1}\right|^{2 \eta}\left|z-z_{2}\right|^{2-2 \eta}\right. \\
\left.-e^{-\nu_{2}}\left|z-z_{1}\right|^{2-2 \eta}\left|z-z_{2}\right|^{2 \eta}\right)^{2} .
\end{gathered}
$$

It is easy to see that $\varphi_{1}$ and $\varphi_{2}$ given by (6.23) and (6.24) have the required singularity (4.5) around $z_{1}$ and $z_{2}$ respectively. In fact each of the functions $\varphi_{1}$ or $\varphi_{2}$ given by (6.23) and (6.24) coincides with the solution describing a saddle point for a two-point function considered in [34]. But in [34] this solution was considered on a full plane with the same parameter $\nu$ everywhere, whereas here each of them is considered on a corresponding halfplane, namely in (6.23) $z$ belongs to the upper half-plane $\Sigma_{1}$, and in (6.24) $z$ belongs to the lower half-plane $\Sigma_{2}$, and we should also remember that, $z_{1} \in \Sigma_{1}$ and $z_{2} \in \Sigma_{2}$. The defect is created by the choice of different parameters $\nu_{1}$ and $\nu_{2}, \nu_{1} \neq \nu_{2}$.

From (6.23) and (6.24) we obtain

$$
\begin{aligned}
\varphi_{1}= & 4 i \pi N_{1}-\log \lambda+2 \log (1-2 \eta) \\
& -2 \log \left(\frac{e^{\nu_{1}}\left|z-z_{1}\right|^{2 \eta}\left|z-z_{2}\right|^{2-2 \eta}}{\left|z_{1}-z_{2}\right|}-\frac{e^{-\nu_{1}}\left|z-z_{1}\right|^{2-2 \eta}\left|z-z_{2}\right|^{2 \eta}}{\left|z_{1}-z_{2}\right|}\right), \\
\varphi_{2}= & 4 i \pi N_{2}-\log \lambda+2 \log (1-2 \eta) \\
& -2 \log \left(-\frac{e^{\nu_{2}}\left|z-z_{1}\right|^{2 \eta}\left|z-z_{2}\right|^{2-2 \eta}}{\left|z_{1}-z_{2}\right|}+\frac{e^{-\nu_{2}}\left|z-z_{1}\right|^{2-2 \eta}\left|z-z_{2}\right|^{2 \eta}}{\left|z_{1}-z_{2}\right|}\right) .
\end{aligned}
$$

Here $N_{1}$ and $N_{2}$ are integer. The possibility to add the term $4 i \pi N_{j}, j=1,2$, results from the invariance of the bulk (2.2) and defect (2.28)-(2.32) Liouville equations of motion under the transformation $\phi_{j} \rightarrow \phi_{j}+2 \pi i N_{j} / b$, or multiplying by $2 b$, under $\varphi_{j} \rightarrow \varphi_{j}+4 \pi i N_{j}$, $j=1,2$. Note that the bulk Liouville equation (2.2) is invariant under the symmetry $\varphi_{j} \rightarrow \varphi_{j}+2 \pi i N_{j}$, and it is broken to $\varphi_{j} \rightarrow \varphi_{j}+4 \pi i N_{j}$ by the exponential terms of the defect action (2.24).

To evaluate the action on the solutions (6.23), (6.24), we will use the strategy used in [20]. Namely we will write the system of differential equations which this action should satisfy. The first equation is (4.11), which given that $\eta_{1}=\eta_{2}=\eta$, reads

$$
b^{2} \frac{\partial S_{\mathrm{cl}}^{\mathrm{def}}}{\partial \eta}=-X_{1}-X_{2} .
$$

where $X_{i}$ is defined in (4.5). The leading terms of $\varphi_{1}$ around $z_{1}$ are

$$
\varphi_{1} \rightarrow-4 \eta \log \left|z-z_{1}\right|+X_{1}
$$

where

$$
X_{1}=4 \pi i N_{1}-\log \lambda+2 \log (1-2 \eta)-(2-4 \eta) \log \left|z_{1}-z_{2}\right|-2 \nu_{1} .
$$

Similarly the leading terms of $\varphi_{2}$ around $z_{2}$ are

$$
\varphi_{2} \rightarrow-4 \eta \log \left|z-z_{2}\right|+X_{2},
$$

where

$$
X_{2}=4 \pi i N_{2}-\log \lambda+2 \log (1-2 \eta)-(2-4 \eta) \log \left|z_{1}-z_{2}\right|+2 \nu_{2} .
$$


Inserting (6.29) and (6.31) in (6.27) one obtains

$$
b^{2} \frac{\partial S_{\mathrm{cl}}^{\mathrm{def}}}{\partial \eta}=-2 \pi i\left(2 N_{1}+2 N_{2}\right)+2 \log \lambda-4 \log (1-2 \eta)+(4-8 \eta) \log \left|z_{1}-z_{2}\right|+2\left(\nu_{1}-\nu_{2}\right) .
$$

We would like to emphasize yet another difference from the calculation of the heavy asymptotic limit of the two-point function in [34]. In the case of the usual two-point function the integers $N_{1}$ and $N_{2}$ are equal since we have one continuous function $\phi$. Here they can be different since we have two different functions $\varphi_{1}$ and $\varphi_{2}$.

The action with defect (4.14) implies also

$$
b^{2} \frac{\partial S_{\mathrm{cl}}^{\mathrm{def}}}{\partial \kappa}=\frac{1}{i \pi} \int_{\partial \Sigma_{1}} e^{\Lambda b} d \tau .
$$

Inserting (6.13) and (6.21) in eq. (2.44) one obtains

$$
e^{\Lambda b}=\frac{1}{2 \sinh \left(\nu_{1}-\nu_{2}\right)} \frac{(2 \eta-1)\left(z_{1}-z_{2}\right)}{\left(z-z_{1}\right)\left(z-z_{2}\right)} .
$$

Using that

$$
\frac{1}{i} \int_{\partial \Sigma_{1}} \frac{d z}{\left(z-z_{1}\right)\left(z-z_{2}\right)}=\frac{2 \pi}{\left(z_{1}-z_{2}\right)}
$$

we obtain

$$
b^{2} \frac{\partial S_{\mathrm{cl}}^{\mathrm{def}}}{\partial \kappa}=\frac{2 \eta-1}{\sinh \left(\nu_{1}-\nu_{2}\right)} .
$$

Integrating equations (6.32) and (6.36) we obtain:

$$
\begin{aligned}
b^{2} S_{N_{1}, N_{2}}^{\text {def }}= & -2 i \pi\left(2 N_{1}+2 N_{2}\right) \eta+4 \eta(1-\eta) \log \left|z_{1}-z_{2}\right| \\
& +2 \eta \log \lambda-(4 \eta-2) \log (1-2 \eta)+4 \eta-\left(\nu_{1}-\nu_{2}\right)(1-2 \eta)+C,
\end{aligned}
$$

where $C$ is a constant. To derive the penultimate term we should remember the relation (6.22). To fix the constant term we can directly compute the action (4.14) for the Ansatz (6.25)-(6.26) with $\eta=0$ :

$$
\begin{aligned}
& \varphi_{1}=4 i \pi N_{1}-\log \lambda-\log \left(\frac{e^{\nu_{1}}}{\left|z_{1}-z_{2}\right|}\left|z-z_{2}\right|^{2}-\frac{e^{-\nu_{1}}}{\left|z_{1}-z_{2}\right|}\left|z-z_{1}\right|^{2}\right)^{2}, \\
& \varphi_{2}=4 i \pi N_{2}-\log \lambda-\log \left(\frac{e^{\nu_{2}}}{\left|z_{1}-z_{2}\right|}\left|z-z_{2}\right|^{2}-\frac{e^{-\nu_{2}}}{\left|z_{1}-z_{2}\right|}\left|z-z_{1}\right|^{2}\right)^{2} .
\end{aligned}
$$

Evaluation of the action (4.14) on the Ansatz (6.38), (6.39) is lengthy and explained in appendix $\mathrm{C}$. The result is

$$
b^{2} S_{0}=2 i \pi\left(N_{1}+N_{2}\right)-\log \lambda-2-\left(\nu_{1}-\nu_{2}\right) .
$$

Comparing (6.40) with (6.37) fixes the constant $C$ :

$$
C=2 i \pi\left(N_{1}+N_{2}\right)-\log \lambda-2 .
$$


Inserting this value of $C$ in (6.37) we indeed obtain (6.10) if we set

$$
\begin{aligned}
& N_{1}=M_{1}, \\
& N_{2}=M_{2} \mp 1,
\end{aligned}
$$

and

$$
2 \pi \sigma=\nu_{1}-\nu_{2}
$$

Some comments are in order at this point:

1. The action (6.37) satisfies the Polyakov relation (4.12) with the accessory parameters defined in (6.17):

$$
b^{2} \frac{\partial S_{\mathrm{cl}}^{\mathrm{def}}}{\partial z_{i}}=(-)^{i+1} \frac{2 \eta(1-\eta)}{z_{1}-z_{2}}, \quad i=1,2 .
$$

2. In eq. (6.4) $M$ takes nonnegative integer values if $\operatorname{Im}(2 \eta-1) / b^{2}>0$, and nonpositive integer values if $\operatorname{Im}(2 \eta-1) / b^{2}<0$. Therefore $N_{1}$ also runs over nonnegative or nonpositive integer values depending on the sign of $\operatorname{Im}(2 \eta-1) / b^{2}$, and $N_{2}$ takes values $\{1,2, \ldots\}$, when $\operatorname{Im}(2 \eta-1) / b^{2}>0$ and $N_{2}$ takes values $\{-1,-2, \ldots\}$, when $\operatorname{Im}(2 \eta-1) / b^{2}<0$. The fact that for the different values of the parameter $\eta$ we should take contribution of different sets of the saddle points is known as the Stokes phenomena [34-37], and was studied in detail for two- and three-point correlation functions of the Liouville field theory in [34]. Recall that it is caused by the fact that the sum (6.4) converges for the different values of $M$ depending on the sign of $\operatorname{Im}(2 \eta-1) / b^{2}$. The values of parameters at which the jump of the set of the contributing saddle point occurs define a (anti-) Stokes line. We have a Stokes line if at some values of parameters the imaginary parts of the actions for two saddle points, say $a$ and $b$, coincide: $\operatorname{Im} S_{a}=\operatorname{Im} S_{b}$. We have an anti-Stokes line if at some values of parameters the real parts of the actions for two saddle points, say $a$ and $b$, coincide: $\operatorname{Re} S_{a}=\operatorname{Re} S_{b}$. Crossing these lines, a jump in the set of the contributing saddle point may occur. For the Stokes lines it is caused by the fact that there is a steepest descent contour connecting two saddle points. For the anti-Stokes line it is implied by the coincidence of the magnitudes of the amplitudes $e^{S_{a}}$ and $e^{S_{b}}$ for the different saddle points. From (6.10) or (6.37) we see that $\operatorname{Re} S_{N_{1}, N_{2}}^{\mathrm{def}}$ are the same for all $N_{1}$ and $N_{2}$ if $\operatorname{Im}(2 \eta-1)=0$. The $\operatorname{line} \operatorname{Im}(2 \eta-1)=0$ is the anti-Stokes line at which indeed we observe a jump in the set of the contributing saddle points.

3. The discussion above of the differences between the calculation of two-point function with and without defect suggests nice interpretation of the defect operator. We have seen that there exist two sources of discontinuity giving rise to the corresponding terms in the defect operators. The heavy asymptotic limit of $D(\alpha)(6.12)$ has an exponential in the numerator and sine function in the denominator. The exponential term in the numerator as we have seen originates from the discontinuity created by the choice of the different parameters $\nu_{1}$ and $\nu_{2}$. The correspondence between the 
$N_{i}$ and $M_{i}$ parameters makes clear that the different logarithmic branch solutions, given by $N_{1}$ and $N_{2}$, are responsible for the quadratic $\sin ^{2} \pi\left(\frac{2 \eta-1}{b^{2}}\right)$ term in the (6.8). On the other hand, as we have mentioned before, in the heavy asymptotic limit the calculation of the usual two-point function one has $N_{1}=N_{2}$, and it reflects the presence of the term $\sin \pi\left(\frac{2 \eta-1}{b^{2}}\right)$ in the denominator of (6.11) in the first degree.

Therefore the denominator $\sin \pi\left(\frac{2 \eta-1}{b^{2}}\right)$ in $D(\alpha)$ reflects the possibility of the choice of different logarithmic branches with $N_{1} \neq N_{2}$ in the solution of the defect equations of motion. The final quantum expression (3.16) results from the quantum corrections restoring $b \leftrightarrow b^{-1}$ duality of the Liouville theory.

Let us analyze in the heavy asymptotic limit also the relation (3.17) between parameter $s$ and $A(b)$

$$
2 \cosh 2 \pi b s=A(b)\left(\frac{W(-b / 2)}{W(0)}\right)^{2} .
$$

It is easy to compute that

$$
\lim _{\mathrm{b} \rightarrow 0} \frac{\mathrm{W}(-\mathrm{b} / 2)}{\mathrm{W}(0)}=-\frac{2}{\sqrt{\lambda}} .
$$

Setting that $s=\frac{\sigma}{b}$, we get

$$
\cosh 2 \pi \sigma=\frac{2 A(0)}{\lambda} .
$$

This implies that the parameter $\kappa$ is proportional to $A(0)$ :

$$
\kappa=\frac{2 A(0)}{\lambda} .
$$

Note that in the light asymptotic limit as well as in the heavy asymptotic limit we get the same relation between $\sigma$ and $\kappa$

$$
\kappa=\cosh 2 \pi \sigma .
$$

\section{Discussion}

The methods developed in this paper can be applied to other theories with defects, like $N=1$ superconformal Liouville theory, conformal and superconformal Toda theories.

The Lagrangian of the $N=1$ Liouville theory with defects is constructed in [14] using the technique of the type II integrable defects. The defect two-point functions in superconformal Liouville theory can as well be constructed via the bootstrap program [45]. It is interesting to use the methods of this paper to construct solutions for superconformal Liouville field theory of the defect equations of motion and study the light and heavy asymptotic limits.

The defect operators in conformal Toda field theory are constructed in $[5,8]$. It is possible using methods of this paper together with the technique of type II defects to construct the Lagrangian of conformal Toda field theory with topological defects and compare with semiclassical limits of defect two-point functions. This program can as well be generalized to superconformal Toda field theory. 
Let us mention also other interesting problems where methods developed in this paper can be applied.

One of the most important problems regarding non-rational conformal field theories is to find for them a relation to a three-dimensional topological field theory description similar to that of the rational ones. This is still a rather difficult and poorly studied problem. The first step was done in [46], where the classical phase space of the ChernSimons gauge theory with $\mathrm{SL}(2, \mathbb{R})$ gauge group has been studied and shown to coincide with the Teichmüller space of Riemann surfaces. It is established by now [47], that the Hilbert space of states obtained by quantizing the Teichmüller space is isomorphic to the space of conformal blocks of Liouville theory. The methods and solutions derived in this paper can be useful to elaborate on the relation between Chern-Simons gauge theory, Teichmüller space of Riemann surfaces, and Liouville field theory including also defects.

Defects appear in many areas in String theory as well as in condensed matter. In particular they play an important role in the entropy entanglement problems [48]. The methods of semiclassical calculations of the defect two-point functions developed here can be used also in that areas. As we mentioned in the introduction heavy and light asymptotic limits appear in many instances of AGT and AdS/CFT correspondences. The insights gained in the study of these limits in the presence of defects can be useful to incorporate defects in these problems.

\section{Acknowledgments}

The work of H.P. was partially supported by the Armenian SCS grant 13-1C132 and by the Armenian-Russian SCS grant-2013. The work of G.S. was partially supported by the Armenian SCS grant 13-1C278 and ICTP Network NET68. We would like also to thank Rubik Poghossian and Shmuel Elitzur for many useful discussions.

\section{A Properties of $\Gamma$ functions}

The limiting behavior of the terms with $\Gamma$ functions can be calculated using the approximation

$$
\Gamma(x) \sim e^{x \log x-x+O(\log x)} .
$$

for $x$ with large positive real part.

For $x$ with negative real part using the formula

$$
\Gamma(x) \Gamma(-x)=-\frac{\pi}{x \sin \pi x},
$$

one can bring problem to the previous case.

We also need the well-known behavior of the $\Gamma(x)$ function for $x$ around zero:

$$
\Gamma(x) \sim \frac{1}{x}
$$




\section{B Volume form on the 3D hyperboloid $\mathrm{H}_{3}^{+}$}

The 3D hyperboloid $H_{3}^{+}$is a pseudo-sphere

$$
X_{0}^{2}-X_{1}^{2}-X_{2}^{2}-X_{3}^{2}=1
$$

in the ambient Minkowski space with the metric:

$$
d s^{2}=-d X_{0}^{2}+d X_{1}^{2}+d X_{2}^{2}+d X_{3}^{2} .
$$

In the parametrization (5.29), one has

$$
\begin{aligned}
X_{0}-X_{1} & =\frac{1}{\rho^{2}}+|w|^{2}, \\
X_{0}+X_{1} & =\rho^{2}, \\
X_{2}+i X_{3} & =\rho w \\
X_{2}-i X_{3} & =\rho \bar{w} .
\end{aligned}
$$

Substituting (B.3) in (B.2) one obtains

$$
d s^{2}=4 \frac{d \rho^{2}}{\rho^{2}}+\rho^{4} d\left(\frac{w}{\rho}\right) d\left(\frac{\bar{w}}{\rho}\right) .
$$

The corresponding volume form is

$$
\sqrt{\operatorname{det} G} d \rho d^{2} w=2 \rho d \rho d^{2} w .
$$

\section{Action evaluation}

The solutions (6.38) and (6.39) have the form:

$$
\begin{aligned}
& \varphi_{1}=4 i \pi N_{1}-\log \lambda-2 \log Z_{1}, \\
& \varphi_{2}=4 i \pi N_{2}-\log \lambda-2 \log Z_{2},
\end{aligned}
$$

where

$$
\begin{aligned}
& Z_{1}=s_{1} z \bar{z}+t_{1} z+u_{1} \bar{z}+v_{1} \\
& Z_{2}=s_{2} z \bar{z}+t_{2} z+u_{2} \bar{z}+v_{2},
\end{aligned}
$$

with

$$
\begin{aligned}
s_{j} & = \pm \frac{2 \sinh \nu_{j}}{\left|z_{1}-z_{2}\right|}, & u_{j} & = \pm \frac{e^{-\nu_{j}} z_{1}-e^{\nu_{j}} z_{2}}{\left|z_{1}-z_{2}\right|}, \\
t_{j} & = \pm \frac{e^{-\nu_{j}} \bar{z}_{1}-e^{\nu_{j}} \bar{z}_{2}}{\left|z_{1}-z_{2}\right|}, & v_{j} & = \pm \frac{e^{\nu_{j}}\left|z_{2}\right|^{2}-e^{-\nu_{j}}\left|z_{1}\right|^{2}}{\left|z_{1}-z_{2}\right|},
\end{aligned}
$$

where we take upper signs for $\nu_{j}$ positive and lower signs for $\nu_{j}$ negative. This choice of signs makes $s_{j} \geq 0$. Note that

$$
s_{j} v_{j}-u_{j} t_{j}=-1 \quad j=1,2 .
$$


It is useful to introduce also real and imaginary parts of $u_{i}$ and $t_{i}$ :

$$
t_{j}=m_{j}+i n_{j}, \quad u_{j}=m_{j}-i n_{j}, \quad j=1,2 .
$$

The function $\tilde{\Lambda}$ can be found setting $\eta=0$ in (6.34)

$$
e^{-\tilde{\Lambda} / 2}=\frac{2 \sinh \left(\nu_{2}-\nu_{1}\right)}{z_{1}-z_{2}}\left(z-z_{1}\right)\left(z-z_{2}\right) .
$$

Before starting the calculations one should examine the zeros of $Z_{1}$ and $Z_{2}$. It is easy to see, that $Z_{j}$ as a quadratic form, vanishes on a circle $C_{j}$ with the center $\left(-\frac{m_{j}}{s_{j}}, \frac{n_{j}}{s_{j}}\right)$ and the radius $\frac{1}{s_{j}}, j=1,2$. Since we have the topological defect, as long as the discs confined by $C_{1}$ and $C_{2}$ do not overlap, we can avoid singularities moving the defect to the safe region between $C_{1}$ and $C_{2}$. Remember that the defect is located along the horizontal axis, and $\varphi_{1}$ and $\varphi_{2}$ are considered on the upper and lower half-planes respectively. Therefore $Z_{1}$ has no zeros if $C_{1}$ is located in the lower half-plane and $Z_{2}$ has no zeros if $C_{2}$ is located in the upper half-plane. This happens, when

$$
n_{1}<-1
$$

and

$$
n_{2}>1 \text {. }
$$

These constraints enable us to avoid the singularities.

Check when these constraints are satisfied. Writing $z_{1}=x_{1}+i y_{1}$, and $z_{2}=x_{2}+i y_{2}$, we get from eq. (C.3):

$$
n_{j}= \pm\left(\frac{e^{\nu_{j}} y_{2}-e^{-\nu_{j}} y_{1}}{\left|z_{2}-z_{1}\right|}\right) .
$$

Recalling that $y_{1}>0$, and $y_{2}<0$, and that we should take upper signs for positive $\nu_{j}$ and lower sign for negative $\nu_{j}$, we see that we obtain negative $n_{1}$ and positive $n_{2}$ taking

$$
\begin{aligned}
& \nu_{1}>0, \quad \text { and } \quad n_{1}=\frac{e^{\nu_{1}} y_{2}-e^{-\nu_{1}} y_{1}}{\left|z_{2}-z_{1}\right|}, \\
& \nu_{2}<0, \quad \text { and } \quad n_{2}=\frac{e^{-\nu_{2}} y_{1}-e^{\nu_{2}} y_{2}}{\left|z_{2}-z_{1}\right|} .
\end{aligned}
$$

Taking $\left|\nu_{j}\right|$ big enough we can always satisfy the condition $\left|n_{j}\right|>1$. This means also that we take in (C.3) the upper sign for $j=1$ and the lower sign $j=2$.

Let us now insert the solution (6.38) and (6.39) in the action (4.14). We will evaluate each term in the $R \rightarrow \infty$ limit. Start by computing the bulk part. The bulk Lagrangians can be written as a total derivative:

$$
\frac{1}{8 \pi i}\left(\partial \varphi_{j} \bar{\partial} \varphi_{j}+4 \lambda e^{\varphi_{j}}\right)=\partial_{\bar{z}} K_{z}^{j}-\partial_{z} K_{\bar{z}}^{j}, \quad j=1,2,
$$

where

$$
K_{z}^{j}=\frac{1}{4 \pi i}\left(-\frac{2}{\left(s_{j} z+u_{j}\right)\left(s_{j}|z|^{2}+t_{j} z+u_{j} \bar{z}+v_{j}\right)}+\frac{s_{j} \log \left(s_{j}|z|^{2}+t_{j} z+u_{j} \bar{z}+v_{j}\right)}{\left(s_{j} z+u_{j}\right)}\right),
$$


and $K_{\bar{z}}^{j}=\bar{K}_{z}^{j}$. We see that under the conditions (C.7) and (C.8) the denominators in (C.13) have no singular points.

The integral over the r.h.s. of (C.12) reduces to the contour integral:

$$
\int_{\Sigma_{j}^{R}}\left(\partial_{\bar{z}} K_{z}^{j}-\partial_{z} K_{\bar{z}}^{j}\right) d^{2} z=\int_{\mathbb{R}} d \tau\left(K_{z}^{j}+K_{\bar{z}}^{j}\right)+\int_{s_{R j}}\left(i K_{z}^{j} z-i K_{\bar{z}}^{j} \bar{z}\right) d \theta
$$

The integral over the semi-circle of the big radius $R$ is evaluated to yield

$$
\int_{s_{R j}}\left(i K_{z}^{j} z-i K_{\bar{z}}^{j} \bar{z}\right) d \theta=\frac{1}{2} \log s_{j} R^{2} .
$$

On the other hand the regularizing terms in the action produce

$$
\begin{aligned}
& \frac{1}{2 \pi} \int_{s_{R 1}} \varphi_{1} d \theta+\log R=-\log \left(s_{1} R^{2}\right)+\log R+2 i \pi N_{1}-\frac{1}{2} \log \lambda, \\
& \frac{1}{2 \pi} \int_{s_{R 2}} \varphi_{2} d \theta+\log R=-\log \left(s_{2} R^{2}\right)+\log R+2 i \pi N_{2}-\frac{1}{2} \log \lambda .
\end{aligned}
$$

The integral over the real axis of the first term in $K^{j}$ gives

$$
-\frac{1}{2 \pi} \int d \tau \frac{2 n_{j}}{\left(s_{j} \tau+u_{j}\right)\left(s_{j} \tau+t_{j}\right)\left(s_{j} \tau^{2}+2 m_{j} \tau+v_{j}\right)}=-\frac{n_{j}}{\sqrt{n_{j}^{2}-1}}+\operatorname{sgn}\left(n_{j}\right)
$$

and of the second produces

$$
\frac{1}{2 \pi} \int d \tau \frac{n_{j} s_{j} \log \left(s_{j} \tau^{2}+2 m_{j} \tau+v_{j}\right)}{\left(s_{j} \tau+u_{j}\right)\left(s_{j} \tau+t_{j}\right)}=-\log \left[\operatorname{sgn}\left(n_{j}\right)\left(n_{j}-\sqrt{n_{j}^{2}-1}\right)\right]-\frac{1}{2} \operatorname{sgn}\left(n_{j}\right) \log s_{j} .
$$

Here we introduced the sign function $\operatorname{sgn}(x) \equiv \frac{x}{\mid x}$.

Remembering that for $\varphi_{1}$ and $\varphi_{2}$ the integrals over the real axis run in the opposite directions, we obtain finally:

$$
\begin{aligned}
& \frac{1}{8 \pi i} \int_{\Sigma_{1}^{R}}\left(\partial \varphi_{1} \bar{\partial} \varphi_{1}+4 \lambda e^{\varphi_{1}}\right) d^{2} z+\frac{1}{8 \pi i} \int_{\Sigma_{2}^{R}}\left(\partial \varphi_{2} \bar{\partial} \varphi_{2}+4 \lambda e^{\varphi_{2}}\right) d^{2} z \\
& +\frac{1}{2 \pi} \int_{s_{R 1}} \varphi_{1} d \theta+\log R+\frac{1}{2 \pi} \int_{s_{R 2}} \varphi_{2} d \theta+\log R= \\
& =-\frac{n_{1}}{\sqrt{n_{1}^{2}-1}}+\frac{n_{2}}{\sqrt{n_{2}^{2}-1}}+\log \left[\frac{n_{2}-\sqrt{n_{2}^{2}-1}}{-n_{1}+\sqrt{n_{1}^{2}-1}}\right] \\
& \quad+2 i \pi N_{1}+2 i \pi N_{2}-\log \lambda-2 .
\end{aligned}
$$

Now we turn to the calculation of the integrals living on the defect. The sum of the last two terms in (4.14), according to the equations of motion (2.28) and (2.29) is

$$
\begin{aligned}
& -\frac{1}{4 \pi i} \int_{-\infty}^{\infty} \mathrm{d} \tau(\partial-\bar{\partial})\left(\varphi_{1}-\varphi_{2}\right)= \\
& =\frac{1}{\pi} \int_{-\infty}^{\infty} \mathrm{d} \tau\left(\frac{n_{1}}{s_{1} \tau^{2}+2 m_{1} \tau+v_{1}}-\frac{n_{2}}{s_{2} \tau^{2}+2 m_{2} \tau+v_{2}}\right)=
\end{aligned}
$$




$$
=\frac{n_{1}}{\sqrt{n_{1}^{2}-1}}-\frac{n_{2}}{\sqrt{n_{2}^{2}-1}} .
$$

We see that (C.21) cancels the first two terms in the third line of (C.20).

Now let us compute the second term on the defect:

$$
\begin{aligned}
& \frac{1}{8 \pi i} \int_{-\infty}^{\infty} \mathrm{d} \tau \tilde{\Lambda}(\partial+\bar{\partial})\left(\varphi_{1}-\varphi_{2}\right)= \\
& =\frac{1}{\pi i} \int_{-\infty}^{\infty} \mathrm{d} \tau \log \left[\left(\tau-z_{1}\right)\left(\tau-z_{2}\right)\right]\left(\frac{s_{1} \tau+m_{1}}{s_{1} \tau^{2}+2 m_{1} \tau+v_{1}}-\frac{s_{2} \tau+m_{2}}{s_{2} \tau^{2}+2 m_{2} \tau+v_{2}}\right) \\
& =\log \left[\frac{-m_{1}+i \sqrt{n_{1}^{2}-1}-z_{2} s_{1}}{m_{1}+i \sqrt{n_{1}^{2}-1}+z_{1} s_{1}}\right]-\log \left[\frac{-m_{2}+i \sqrt{n_{2}^{2}-1}-z_{2} s_{2}}{m_{2}+i \sqrt{n_{2}^{2}-1}+z_{1} s_{2}}\right] .
\end{aligned}
$$

To simplify this expression one can show, introducing an angle $e^{i \xi}=\frac{z_{2}-z_{1}}{\left|z_{2}-z_{1}\right|}$, that

$$
\begin{aligned}
-m_{j}+i \sqrt{n_{j}^{2}-1}-z_{2} s_{j} & =i\left((-)^{j} i e^{-\nu_{j}} e^{i \xi}-n_{j}+\sqrt{n_{j}^{2}-1}\right) \\
m_{j}+i \sqrt{n_{j}^{2}-1}+z_{1} s_{j} & =i\left((-)^{j+1} i e^{\nu_{j}} e^{i \xi}+n_{j}+\sqrt{n_{j}^{2}-1}\right), \quad j=1,2 .
\end{aligned}
$$

We can also prove

$$
\begin{array}{r}
\left((-)^{j} i e^{-\nu_{j}} e^{i \xi}-n_{j}+\sqrt{n_{j}^{2}-1}\right)\left((-)^{j+1} i e^{\nu_{j}} e^{-i \xi}-n_{j}+\sqrt{n_{j}^{2}-1}\right) \\
=(-)^{j}\left(-n_{j}+\sqrt{n_{j}^{2}-1}\right)\left(i e^{-\nu_{j}} e^{i \xi}-i e^{\nu_{j}} e^{-i \xi}-(-)^{j} 2 n_{j}\right)
\end{array}
$$

and writing $z_{1}=x_{1}+i y_{1}$ and $z_{2}=x_{2}+i y_{2}$, one obtains that

$$
\left(i e^{-\nu_{j}} e^{i \xi}-i e^{\nu_{j}} e^{-i \xi}-2(-)^{j} n_{j}\right)=-\frac{2 i \sinh \nu_{j}}{\left|z_{2}-z_{1}\right|}\left(x_{2}-x_{1}+i\left(y_{2}+y_{1}\right)\right) .
$$

And finally we need

$$
\begin{aligned}
& \left((-)^{j+1} i e^{\nu_{j}} e^{-i \xi}-n_{j}+\sqrt{n_{j}^{2}-1}\right)\left((-)^{j+1} i e^{\nu_{j}} e^{i \xi}+n_{j}+\sqrt{n_{j}^{2}-1}\right) \\
& =-2 e^{\nu_{j}}\left(\cosh \nu_{j}+(-)^{j} i \cos \xi \sqrt{n_{j}^{2}-1}+(-)^{j} n_{j} \sin \xi\right) .
\end{aligned}
$$

Using all these identities, and noting that the terms in the r.h.s. of (C.25), independent on $j$, get canceled, we obtain

$$
\begin{aligned}
& \frac{1}{8 \pi i} \int_{-\infty}^{\infty} \mathrm{d} \tau \tilde{\Lambda}(\partial+\bar{\partial})\left(\varphi_{1}-\varphi_{2}\right)= \\
& =\log \left[\frac{\left(\cosh \nu_{2}+i \cos \xi \sqrt{n_{2}^{2}-1}+n_{2} \sin \xi\right) \sinh \nu_{1}\left(-n_{1}+\sqrt{n_{1}^{2}-1}\right)}{\left(\cosh \nu_{1}-i \cos \xi \sqrt{n_{1}^{2}-1}-n_{1} \sin \xi\right) \sinh \nu_{2}\left(n_{2}-\sqrt{n_{2}^{2}-1}\right)}\right]+\nu_{2}-\nu_{1} .
\end{aligned}
$$

The third multipliers in the numerator and in the denominator of the argument of the logarithm in (C.27) together cancel the third term in the third line of (C.20). It is easy 
to see that the remaining logarithmic term after this cancellation is a pure argument since the remaining numerator and denominator have the same modulus:

$$
\frac{1}{\sinh ^{2} \nu_{j}}\left[\left(\cosh \nu_{j}+(-)^{j} n_{j} \sin \xi\right)^{2}+\left(n_{j}^{2}-1\right) \cos ^{2} \xi\right]=\frac{\left(x_{1}-x_{2}\right)^{2}+\left(y_{1}+y_{2}\right)^{2}}{\left|z_{1}-z_{2}\right|^{2}}
$$

And finally the first integral on the defect is

$$
\begin{aligned}
- & \frac{1}{16 \pi i} \int_{-\infty}^{\infty} \mathrm{d} \tau\left(\varphi_{2} \partial_{\tau} \varphi_{1}-\varphi_{1} \partial_{\tau} \varphi_{2}\right)= \\
= & -\frac{1}{2 \pi i} \int_{-\infty}^{\infty} \mathrm{d} \tau\left[\log \left(s_{2} \tau^{2}+2 m_{2} \tau+v_{2}\right) \frac{s_{1} \tau+m_{1}}{s_{1} \tau^{2}+2 m_{1} \tau+v_{1}}\right. \\
& \left.-\log \left(s_{1} \tau^{2}+2 m_{1} \tau+v_{1}\right) \frac{s_{2} \tau+m_{2}}{s_{2} \tau^{2}+2 m_{2} \tau+v_{2}}\right]= \\
= & \log \left[\frac{-s_{1} m_{2}+s_{2} m_{1}+i\left(s_{2} \sqrt{n_{1}^{2}-1}+s_{1} \sqrt{n_{2}^{2}-1}\right)}{s_{1} m_{2}-s_{2} m_{1}+i\left(s_{2} \sqrt{n_{1}^{2}-1}+s_{1} \sqrt{n_{2}^{2}-1}\right)}\right] .
\end{aligned}
$$

Obviously this is also a pure argument. After cumbersome but straightforward calculation one can show that:

$$
\frac{\left(-s_{1} m_{2}+s_{2} m_{1}+i\left(s_{2} \sqrt{n_{1}^{2}-1}+s_{1} \sqrt{n_{2}^{2}-1}\right)\right)}{\left(s_{1} m_{2}-s_{2} m_{1}+i\left(s_{2} \sqrt{n_{1}^{2}-1}+s_{1} \sqrt{n_{2}^{2}-1}\right)\right.} \frac{\left(\cosh \nu_{2}+i \cos \xi \sqrt{n_{2}^{2}-1}+n_{2} \sin \xi\right) \sinh \nu_{1}}{\left(\cosh \nu_{1}-i \cos \xi \sqrt{n_{1}^{2}-1}-n_{1} \sin \xi\right) \sinh \nu_{2}}=1
$$

and therefore (C.29) cancels the remaining logarithmic terms in (C.27). Collecting all we obtain:

$$
b^{2} S_{0}=2 i \pi\left(N_{1}+N_{2}\right)-\log \lambda-2+\nu_{2}-\nu_{1}
$$

\section{Defect two-point function}

First let us briefly explain how to derive the Cardy-Lewellen cluster condition for defects in rational theories without multiplicities $[7,9]$. Suppose we have a two-dimensional rational conformal field theory with primary fields $\Phi_{i}$. The vacuum state is attributed $i=0$. A topological defect $X$ is a sum of projectors

$$
X=\sum_{i} \mathcal{D}^{i} P^{i}
$$

where

$$
P^{i}=\sum_{N, \bar{N}}(|i, N\rangle \otimes|i, \bar{N}\rangle)(\langle i, N| \otimes\langle i, \bar{N}|)
$$

Here $|i, N\rangle$ and $|i, \bar{N}\rangle$ are vectors of orthonormal bases of left and right copies of the highest weight representations $R_{i}$ respectively. Two-point functions with a defect $X$ insertion can be written as

$$
\left\langle\Phi_{i}\left(z_{1}, \bar{z}_{1}\right) X \Phi_{i}\left(z_{2}, \bar{z}_{2}\right)\right\rangle=\frac{D^{i}}{\left(z_{1}-z_{2}\right)^{2 \Delta_{i}}\left(\bar{z}_{1}-\bar{z}_{2}\right)^{2 \Delta_{i}}},
$$

where

$$
D^{i}=\mathcal{D}^{i} C_{i i}
$$

and $C_{i i}$ is a two-point function. 
The fields $\Phi_{i}$ via the operator product expansion (OPE) form an algebra with structure constant $C_{i j}^{k}[49,50]$ :

$$
\Phi_{i}\left(z_{1}, \bar{z}_{1}\right) \Phi_{j}\left(z_{2}, \bar{z}_{2}\right)=\sum_{k} \frac{C_{i j}^{k}}{\left(z_{1}-z_{2}\right)^{\Delta_{i}+\Delta_{j}-\Delta_{k}}\left(\bar{z}_{1}-\bar{z}_{2}\right)^{\Delta_{i}+\Delta_{j}-\Delta_{k}}} \Phi_{k}\left(z_{2}, \bar{z}_{2}\right)+\text { descendants } .
$$

We need also to introduce the fusion number $N_{i j}^{k}$. This is the number of occurrence of the field $\Phi_{k}$ in the operator product expansion of $\Phi_{i}$ and $\Phi_{j}$. Here we assume that $N_{i j}^{k}$ takes two values: 0 and 1 . Consider the following four-point correlation function with the defects insertions on a torus:

$$
\left\langle\Phi_{j}\left(z_{1}, \bar{z}_{1}\right) \Phi_{i}\left(z_{2}, \bar{z}_{2}\right) X \Phi_{i}\left(z_{3}, \bar{z}_{3}\right) \Phi_{j}\left(z_{4}, \bar{z}_{4}\right) X\right\rangle .
$$

Using (D.5) and (D.3) one can compute (D.6) in two pictures. In the first picture at the beginning we use OPE (D.5) for the pairs $\Phi_{j}\left(z_{1}, \bar{z}_{1}\right) \Phi_{i}\left(z_{2}, \bar{z}_{2}\right)$ and $\Phi_{i}\left(z_{3}, \bar{z}_{3}\right) \Phi_{j}\left(z_{4}, \bar{z}_{4}\right)$ and then (D.3) for the fields produced in this process. This results in

$$
\sum_{k} D^{k} D^{0}\left(C_{i j}^{k} \mathcal{F}_{k}\left[\begin{array}{ll}
i & i \\
j & j
\end{array}\right]\right)^{2}
$$

where $\mathcal{F}_{k}\left[\begin{array}{ll}i & i \\ j & j\end{array}\right]$ is the so called conformal block [49, 50] giving the contribution of the descendant fields in the OPE (D.5). It appears squared since it is separately produced by the left and right modes.

In the second picture we move the field $\Phi_{j}\left(z_{1}, \bar{z}_{1}\right)$ to the rightmost position:

$$
\left\langle\Phi_{i}\left(z_{2}, \bar{z}_{2}\right) X \Phi_{i}\left(z_{3}, \bar{z}_{3}\right) \Phi_{j}\left(z_{4}, \bar{z}_{4}\right) X \Phi_{j}\left(z_{1}, \bar{z}_{1}\right)\right\rangle
$$

and then use twice (D.3) resulting in

$$
D^{i} D^{j}\left(\mathcal{F}_{0}\left[\begin{array}{ll}
i & j \\
i & j
\end{array}\right]\right)^{2}+\cdots
$$

Using the fusing matrix:

$$
\mathcal{F}_{k}\left[\begin{array}{cc}
i & i \\
j & j
\end{array}\right]=\sum_{m} F_{k m}\left[\begin{array}{cc}
j & j \\
i & i
\end{array}\right] \mathcal{F}_{m}\left[\begin{array}{ll}
i & j \\
i & j
\end{array}\right]
$$

we obtain

$$
\sum_{k} D^{0} D^{k}\left(C_{i j}^{k} F_{k 0}\left[\begin{array}{cc}
j & j \\
i & i
\end{array}\right]\right)^{2}=D^{i} D^{j} .
$$

This is the Cardy-Lewellen cluster condition for defects.

Using that for rational conformal field theory the structure constants and the fusion matrix satisfy the relation [51]

$$
C_{i j}^{p} F_{p, 0}\left[\begin{array}{cc}
j & j \\
i & i
\end{array}\right]=\frac{\xi_{i} \xi_{j}}{\xi_{0} \xi_{p}}
$$


where

$$
\xi_{i}=\sqrt{C_{i i} F_{i}},
$$

and

$$
F_{i} \equiv F_{0,0}\left[\begin{array}{ll}
i & i \\
i & i
\end{array}\right],
$$

the Cardy-Lewellen condition for defects (D.11) simplifies to

$$
\sum_{k} D^{0} D^{k} N_{i j}^{k}\left(\frac{\xi_{i} \xi_{j}}{\xi_{0} \xi_{k}}\right)^{2}=D^{i} D^{j}
$$

Define $\Psi^{k}$ as

$$
\frac{D^{k}}{D^{0}}=\Psi^{k}\left(\frac{\xi^{k}}{\xi^{0}}\right)^{2}
$$

eq. (D.15) becomes the following equation for $\Psi^{k}$

$$
\sum_{k} \Psi^{k} N_{i j}^{k}=\Psi^{i} \Psi^{j}
$$

And finally to find the coefficient $\mathcal{D}^{i}$ of the defects expansion to projectors we should, according to (D.4), divide $D^{i}$ by the two-point function.

Let us now apply this machinery to the Liouville theory. Liouville theory is a nonrational theory, but we can overcome the difficulties caused by the infinite number of primaries. First of all it is shown in [8] that the relation (D.12) works also in diagonal non-rational theories. In particular it is shown in [8] that in the Liouville theory

$$
\xi(\alpha)=\frac{\sqrt{W(0) W(Q)}}{W(\alpha)} .
$$

and (D.12) takes the form:

$$
C_{\alpha_{1}, \alpha_{2}}^{\alpha_{3}} F_{\alpha_{3}, 0}\left[\begin{array}{ll}
\alpha_{1} & \alpha_{1} \\
\alpha_{2} & \alpha_{2}
\end{array}\right]=W(0) \frac{W\left(\alpha_{3}\right)}{W\left(\alpha_{1}\right) W\left(\alpha_{2}\right)},
$$

where $W(\alpha)$ is ZZ function (3.7). The second problem is that in the Liouville theory the OPE of primary fields with generic $\alpha_{1}$, and $\alpha_{2}$ contains infinite number of intermediate primary states, which makes the use of the equation (D.17) rather problematic. This difficulty can be resolved via Teschner's trick [52]. Teschner's tricks relies on the existence of degenerate fields in the Liouville field theory. The fields $V_{\alpha}$ with $\alpha$ belonging to the set

$$
\alpha_{m, n}=\frac{1-m}{2 b}+\frac{1-n}{2} b, \quad m, n \in \mathbb{N}
$$

produce in the OPE with other fields just a finite number of the fields. Teschner's trick suggests to take as $\Phi_{j}$ one of the fields $V_{\alpha_{m, n}}$. This choice will yield only finite number of terms in the l.h.s. of (D.17). The simplest of the fields (D.20) is $V_{-b / 2}$. With a generic field $V_{\alpha}$ it has the OPE:

$$
V_{\alpha} V_{-b / 2} \sim C_{-b / 2, \alpha}^{\alpha-b / 2} V_{\alpha-b / 2}+C_{-b / 2, \alpha}^{\alpha+b / 2} V_{\alpha+b / 2}
$$


With $j=-\frac{b}{2}, i=\alpha$, and $k=\alpha \pm b / 2$, the equations (D.17) and (D.16) take the form:

$$
\Psi(\alpha) \Psi(-b / 2)=\Psi(\alpha-b / 2)+\Psi(\alpha+b / 2),
$$

and

$$
\frac{D(\alpha)}{D(0)}=\Psi(\alpha)\left(\frac{W(0)}{W(\alpha)}\right)^{2}
$$

The solution of the equation (D.22) is

$$
\Psi_{m, n}(\alpha)=\frac{\sin \left(\pi m b^{-1}(2 \alpha-Q)\right) \sin (\pi n b(2 \alpha-Q))}{\sin \left(\pi m b^{-1} Q\right) \sin (\pi n b Q)},
$$

Using (D.23) we obtain for the defect two-point function

$$
D_{m, n}(\alpha)=-\frac{2 \sqrt{2} \sin \left(\pi m b^{-1}(2 \alpha-Q)\right) \sin (\pi n b(2 \alpha-Q))}{W^{2}(\alpha)} .
$$

And finally dividing on $S(\alpha)(3.5)$ we get

$$
\mathcal{D}_{m, n}(\alpha)=\frac{\sin \left(\pi m b^{-1}(2 \alpha-Q)\right) \sin (\pi n b(2 \alpha-Q))}{\sin \pi b^{-1}(2 \alpha-Q) \sin \pi b(2 \alpha-Q)} .
$$

Note that the defect given by $(m, n)=(1,1)$ is the identity defect.

But this is not the end of the story. Let us now explain how to obtain two-point function for the continuous family of defects. We will use the strategy developed in $[23,53]$ in the context of the Liouville and Toda theories with a boundary. Assume that we have a family of defects parameterized by $\kappa$. In this case $D(-b / 2)$, which is the two-point function of the degenerate field $V_{-b / 2}$ in the presence of defect, will be a function of $\kappa$ and $b$. Denote the ratio $D(-b / 2) / D(0)$ by $A(\kappa, b)$ and define

$$
D(\alpha)=\frac{\tilde{\Psi}(\alpha)}{W^{2}(\alpha)} .
$$

Substituting $A(\kappa, b)$ and $\tilde{\Psi}(\alpha)$ in (D.15) again for $j=-\frac{b}{2}, i=\alpha$, and $k=\alpha \pm b / 2$, we obtain a linear equation for $\tilde{\Psi}(\alpha)$ :

$$
\left(\frac{W(-b / 2)}{W(0)}\right)^{2} A \tilde{\Psi}(\alpha)=\tilde{\Psi}(\alpha-b / 2)+\tilde{\Psi}(\alpha+b / 2) .
$$

The solution of (D.28) is indeed a one-parametric family,

$$
\tilde{\Psi}_{s}(\alpha)=-2^{1 / 2} \cosh (2 \pi s(2 \alpha-Q)),
$$

with a parameter $s$ related to $A$ by

$$
2 \cosh 2 \pi b s=A\left(\frac{W(-b / 2)}{W(0)}\right)^{2}
$$

Substituting (D.29) in (D.27) we obtain for $D_{s}(\alpha)$ and $\mathcal{D}_{s}(\alpha)$ respectively

$$
D_{s}(\alpha)=-\frac{2^{1 / 2} \cosh (2 \pi s(2 \alpha-Q))}{W^{2}(\alpha)} .
$$




$$
\mathcal{D}_{s}(\alpha)=\frac{\cosh (2 \pi s(2 \alpha-Q))}{2 \sin \pi b^{-1}(2 \alpha-Q) \sin \pi b(2 \alpha-Q)} .
$$

We would like to finish by a remark on the world-volume of the defects (D.26) and (D.32). Recall the notion of the defect world-volume [54].

The values of the Liouville fields $\phi_{1}$ and $\phi_{2}$ on a point $\tau$ of the defect line form a point $\left(\phi_{1}(\tau), \phi_{2}(\tau)\right)$ in the plane $\mathbb{R}^{2}$. The set of all such points may be restricted to belong to a submanifold $Q$ of the plane $\mathbb{R}^{2}$, depending on the defect condition. The submanifold $Q$ is called the world-volume of the defect. It can be shown that the world-volume of the defects (D.32) coincide with all $\mathbb{R}^{2}$, which means that there are no constraints on the values of the fields $\phi_{1}$ and $\phi_{2}$. But the world-volume of the defects (D.26) is a one-dimensional. It can be easily seen for the identity defect $\mathcal{D}_{1,1}$, since for the identity defect there is no discontinuity in the value of Liouville field and therefore $\phi_{1}$ and $\phi_{2}$ satisfy $\phi_{1}(\tau)=\phi_{2}(\tau)$ in any point $\tau$ of the defect line.

Open Access. This article is distributed under the terms of the Creative Commons Attribution License (CC-BY 4.0), which permits any use, distribution and reproduction in any medium, provided the original author(s) and source are credited.

\section{References}

[1] V.B. Petkova and J.B. Zuber, Generalized twisted partition functions, Phys. Lett. B 504 (2001) 157 [hep-th/0011021] [INSPIRE].

[2] L.F. Alday, D. Gaiotto, S. Gukov, Y. Tachikawa and H. Verlinde, Loop and surface operators in $N=2$ gauge theory and Liouville modular geometry, JHEP 01 (2010) 113 [arXiv:0909.0945] [INSPIRE].

[3] N. Drukker, J. Gomis, T. Okuda and J. Teschner, Gauge theory loop operators and Liouville theory, JHEP 02 (2010) 057 [arXiv:0909.1105] [INSPIRE].

[4] V.B. Petkova, On the crossing relation in the presence of defects, JHEP 04 (2010) 061 [arXiv: 0912.5535] [INSPIRE].

[5] N. Drukker, D. Gaiotto and J. Gomis, The virtue of defects in $4 D$ gauge theories and $2 D$ CFTs, JHEP 06 (2011) 025 [arXiv: 1003.1112] [INSPIRE].

[6] J. Fuchs, C. Schweigert and C. Stigner, The classifying algebra for defects, Nucl. Phys. B 843 (2011) 673 [arXiv: 1007.0401] [INSPIRE].

[7] G. Sarkissian, Defects and permutation branes in the Liouville field theory, Nucl. Phys. B 821 (2009) 607 [arXiv:0903.4422] [INSPIRE].

[8] G. Sarkissian, Some remarks on D-branes and defects in Liouville and Toda field theories, Int. J. Mod. Phys. A 27 (2012) 1250181 [arXiv:1108.0242] [INSPIRE].

[9] V.B. Petkova and J.B. Zuber, The many faces of Ocneanu cells, Nucl. Phys. B 603 (2001) 449 [hep-th/0101151] [INSPIRE].

[10] P. Bowcock, E. Corrigan and C. Zambon, Classically integrable field theories with defects, Int. J. Mod. Phys. A 19S2 (2004) 82 [hep-th/0305022] [INSPIRE].

[11] P. Bowcock, E. Corrigan and C. Zambon, Affine Toda field theories with defects, JHEP 01 (2004) 056 [hep-th/0401020] [INSPIRE]. 
[12] E. Corrigan and C. Zambon, A new class of integrable defects, J. Phys. A 42 (2009) 475203 [arXiv:0908.3126] [INSPIRE].

[13] A.R. Aguirre, J.F. Gomes, N.I. Spano and A.H. Zimerman, Type-II super-Bäcklund transformation and integrable defects for the $N=1$ super sinh-Gordon model, JHEP 06 (2015) 125 [arXiv: 1504.07978] [INSPIRE].

[14] A.R. Aguirre, Type-II defects in the super-Liouville theory, J. Phys. Conf. Ser. 474 (2013) 012001 [arXiv:1312.3463] [InSPIRE].

[15] V. Caudrelier, On a systematic approach to defects in classical integrable field theories, Int. J. Geom. Meth. Mod. Phys. 5 (2008) 1085 [arXiv:0704.2326] [INSPIRE].

[16] V. Caudrelier, Multisymplectic approach to integrable defects in the sine-Gordon model, J. Phys. A 48 (2015) 195203 [arXiv:1411.5171] [inSPIRE].

[17] C. Robertson, Defect fusing rules in affine Toda field theory, J. Phys. A 47 (2014) 485205 [arXiv: 1408.1960] [INSPIRE].

[18] J. Avan and A. Doikou, Liouville integrable defects: the non-linear Schrödinger paradigm, JHEP 01 (2012) 040 [arXiv:1110.4728] [INSPIRE].

[19] N. Seiberg, Notes on quantum Liouville theory and quantum gravity, Prog. Theor. Phys. Suppl. 102 (1990) 319 [inSPIRE].

[20] A.B. Zamolodchikov and A.B. Zamolodchikov, Structure constants and conformal bootstrap in Liouville field theory, Nucl. Phys. B 477 (1996) 577 [hep-th/9506136] [INSPIRE].

[21] P.H. Ginsparg and G.W. Moore, Lectures on 2D gravity and 2D string theory (TASI 1992), in Proceedings, Recent directions in particle theory, LA-UR-92-3479, Boulder U.S.A. (1992), pg. 277 [hep-th/9304011] [INSPIRE].

[22] V.A. Fateev and A.V. Litvinov, Correlation functions in conformal Toda field theory. I, JHEP 11 (2007) 002 [arXiv:0709.3806] [INSPIRE].

[23] V. Fateev and S. Ribault, Conformal Toda theory with a boundary, JHEP 12 (2010) 089 [arXiv: 1007.1293] [INSPIRE].

[24] L. Hadasz and Z. Jaskolski, Semiclassical limit of the FZZT Liouville theory, Nucl. Phys. B 757 (2006) 233 [hep-th/0603164] [INSPIRE].

[25] P. Menotti and E. Tonni, Liouville field theory with heavy charges. I. The pseudosphere, JHEP 06 (2006) 020 [hep-th/0602206] [INSPIRE].

[26] P. Menotti and E. Tonni, Liouville field theory with heavy charges. II. The conformal boundary case, JHEP 06 (2006) 022 [hep-th/0602221] [INSPIRE].

[27] A. Mironov and A. Morozov, Proving AGT relations in the large-c limit, Phys. Lett. B 682 (2009) 118 [arXiv:0909.3531] [INSPIRE].

[28] V. Fateev and S. Ribault, The large central charge limit of conformal blocks, JHEP 02 (2012) 001 [arXiv:1109.6764] [INSPIRE].

[29] N. Hama and K. Hosomichi, AGT relation in the light asymptotic limit, JHEP 10 (2013) 152 [arXiv: 1307.8174] [INSPIRE].

[30] M. Piatek, Classical torus conformal block, $N=2^{*}$ twisted superpotential and the accessory parameter of Lamé equation, JHEP 03 (2014) 124 [arXiv: 1309.7672] [INSPIRE]. 
[31] E. Hijano, P. Kraus, E. Perlmutter and R. Snively, Semiclassical Virasoro blocks from $A d S_{3}$ gravity, arXiv: 1508.04987 [INSPIRE].

[32] K.B. Alkalaev and V.A. Belavin, Classical conformal blocks via AdS/CFT correspondence, JHEP 08 (2015) 049 [arXiv: 1504.05943] [INSPIRE].

[33] A.L. Fitzpatrick, J. Kaplan and M.T. Walters, Virasoro conformal blocks and thermality from classical background fields, arXiv:1501.05315 [INSPIRE].

[34] D. Harlow, J. Maltz and E. Witten, Analytic continuation of Liouville theory, JHEP 12 (2011) 071 [arXiv:1108.4417] [INSPIRE].

[35] E. Witten, Analytic continuation of Chern-Simons theory, AMS/IP Stud. Adv. Math. 50 (2011) 347 [arXiv: 1001.2933] [INSPIRE].

[36] M. Mariño, Lectures on non-perturbative effects in large- $N$ gauge theories, matrix models and strings, Fortsch. Phys. 62 (2014) 455 [arXiv:1206.6272] [INSPIRE].

[37] S. Pasquetti and R. Schiappa, Borel and Stokes nonperturbative phenomena in topological string theory and $c=1$ matrix models, Annales Henri Poincaré 11 (2010) 351 [arXiv: 0907.4082] [INSPIRE].

[38] J. Liouville, Sur l'équation aux dérivées partielles $((\partial 2 \log \lambda) \partial u \partial u) \pm 2 \lambda a 2=0$ (in French), $J$. Math. Pures Appl. 18 (1853) 71.

[39] J.-L. Gervais and A. Neveu, The dual string spectrum in Polyakov's quantization. 1, Nucl. Phys. B 199 (1982) 59 [inSPIRE].

[40] A.B. Zamolodchikov and A.B. Zamolodchikov, Liouville field theory on a pseudosphere, hep-th/0101152 [INSPIRE].

[41] A. Recknagel, Permutation branes, JHEP 04 (2003) 041 [hep-th/0208119] [InSPIRE].

[42] P. Zograf and L.A. Takhtajan, On Liouville's equation, accessory parameters, and the geometry of Teichmüller space for Riemann surfaces of genus 0 , Math. USSR Sbornik 60 (1988) 143.

[43] S. Weinberg, The quantum theory of fields. Vol. 1: foundations, Cambridge Univ. Pr., Cambridge U.K. (1995).

[44] W.G.C. Boyd, Gamma function asymptotics by an extensions of the methods of steepest descent, Proc. Roy. Soc. London A 447 (1994) 609.

[45] H. Poghosyan and G. Sarkissian, work in progress.

[46] H.L. Verlinde, Conformal field theory, $2 D$ quantum gravity and quantization of Teichmüller space, Nucl. Phys. B 337 (1990) 652 [INSPIRE].

[47] J. Teschner, From Liouville theory to the quantum geometry of Riemann surfaces, hep-th/0308031 [INSPIRE].

[48] P. Calabrese and J.L. Cardy, Entanglement entropy and quantum field theory, J. Stat. Mech. 06 (2004) P06002 [hep-th/0405152] [InSPIRE].

[49] A.A. Belavin, A.M. Polyakov and A.B. Zamolodchikov, Infinite conformal symmetry in two-dimensional quantum field theory, Nucl. Phys. B 241 (1984) 333 [INSPIRE].

[50] P. Di Francesco, P. Mathieu and D. Senechal, Conformal field theory, Graduate Texts in Contemporary Physics, Springer-Verlag, New York U.S.A. (1997). 
[51] R.E. Behrend, P.A. Pearce, V.B. Petkova and J.-B. Zuber, Boundary conditions in rational conformal field theories, Nucl. Phys. B 570 (2000) 525 [Corrigendum ibid. B 579 (2000) 707] [hep-th/9908036] [INSPIRE].

[52] J. Teschner, On the Liouville three point function, Phys. Lett. B 363 (1995) 65 [hep-th/9507109] [INSPIRE].

[53] V. Fateev, A.B. Zamolodchikov and A.B. Zamolodchikov, Boundary Liouville field theory. 1. Boundary state and boundary two point function, hep-th/0001012 [INSPIRE].

[54] J. Fuchs, C. Schweigert and K. Waldorf, Bi-branes: target space geometry for world sheet topological defects, J. Geom. Phys. 58 (2008) 576 [hep-th/0703145] [InSPIRE]. 